\title{
CAMBIO DE RUMBO. INTERACCIÓN COMERCIAL ENTRE EL BRONCE FINAL ATLÁNTICO IBÉRICO Y MICÉNICO EN EL MEDITERRÁNEO CENTRAL (1425-1050 A.C.)
}

\author{
CHANGING THE ROUTE. TRADE \\ INTERACTION BETWEEN THE ATLANTIC \\ IBERIAN LATE BRONZE AGE AND THE \\ MYCENAEAN IN THE CENTRAL \\ MEDITERRANEAN (1425-1050 BC)
}

ALFREDO MEDEROS MARTÍN (*)

\section{RESUMEN}

El objetivo de este trabajo es ofrecer una nueva interpretación sobre los intercambios comerciales durante el Bronce Final IC-IIC, $c a$. 1425-1050 AC, entre el Mediterráneo Occidental y Central, a partir de una revisión de dos tipos de artefactos metálicos, hachas y espadas, procedentes de la Península Ibérica, y presentes en Cerdeña, Sicilia y el Lazio en la Península Italiana. Se parte de tres premisas; la primera, la cronología calibrada del Bronce Final en la Península Ibérica, Cerdeña y Sicilia-Eolias. En segundo lugar, un encuadre temporal más preciso de los artefactos ibéricos presentes en los yacimientos y depósitos italianos. Y finalmente, el análisis de la confluencia del comercio atlántico y micénico en el Mediterráneo Central, particularmente durante el Bronce Final IC-IIA/ Heládico Final IIIA-IIIB, ca. 1425-1225 AC, para poner en evidencia que la presencia de cerámicas micénicas en Andalucía no puede tratarse de un fenómeno aislado, sino que se integra dentro de la interrelación comercial que existió entre el Mediterráneo Occidental y Oriental alrededor de las islas de Cerdeña y Sicilia del Mediterráneo Central.

(*) Becario Postdoctoral de la Dirección General de Investigación Científica y Técnica en el Institute of Archaeology, Oxford University, 36, Beaumont Street, Oxford OX12PG. Tel. 4418652782 40. Fax 4418652782 54. Correo electrónico: Alfredo.Mederos@ archaeology.oxford.ac.uk.

El artículo fue remitido en su versión final el 27-XI-1997.

\begin{abstract}
The aim of this work is to present a new interpretation on the commercial exchanges during the Late Bronze Age IC-IIC, c. 1425-1050 BC, between the Western and Central Mediterranean, starting from a review of two types of metallic artefact, axes and swords, originating from the Iberian Peninsula, and present in Sardinia, Sicily and Lazio in the Italian Peninsula. We start from three premises: the calibrated chronology of the Late Bronze Age in the Iberian Peninsula, Sardinia and Sicily-Eolian. Secondly, a more accurate chronology of the Iberian artefacts present in Italian settlements and deposits. And finally, the analysis of the confluence between the Atlantic and Mycenaean trade in the Central Mediterranean, particularly during the Late Bronze Age IC-IIA/Late Helladic IIIA-IIIB, $c$. 1425-1225 BC, to reveal that the mycenaean pottery presence in Andalusia can not be tried as an isolated phenomenon, but is integrated within the commercial interrelationship that existed between the Western and Eastern Mediterranean around the islands of Sardinia and Sicily in the Central Mediterranean.
\end{abstract}

Palabras clave: Sistemas Mundiales. Bronce Final. Península Ibérica. Comercio. Artefactos metálicos. Cerámica micénica. Cerdeña. Sicilia. Península Italiana.

Key words: World Systems. Late Bronze Age. Iberian Peninsula. Trade. Metallic artefacts. Mycenaean pottery. Sardinia. Sicily. Italian Peninsula. 


\section{INTRODUCCIÓN}

La presencia de metalurgia atlántica en el Mediterráneo Central es un tema recurrente en la bibliografía desde la publicación por Taramelli (1915) del depósito de Monte Sa Idda en Cerdeña, que con el antecedente del depósito de Venat (George y Chauvet, 1894; Coffyn et alii, 1981) y la posterior aparición del depósito o pecio de Huelva (Almagro Basch, 1940 y 1958; Ruiz-Gálvez, 1995), posibilitaba la interrelación de los tres depósitos para documentar la presencia de relaciones entre la fachada atlántica de Francia y la Península Ibérica durante el Bronce Final hacia el Mediterráneo Central.

Dichos intercambios, según la valoración tradicional, se habrían producido en un momento tardío. Inicialmente, para el presunto naufragio del pecio de Huelva, Almagro Basch (1940: 142) propuso la fecha del 750 a.C. Tras disponerse de la serie de dataciones del depósito de la ría de Huelva, ca. 875-850 a.C. (Almagro Gorbea, 1977: 524-525) incluso se llegó a seguir manteniendo fechas aún más recientes, caso de Coffyn (1985: 274) que señaló el 750-700 a.C. Otros investigadores, por el contrario, las aceptaron proponiendo un 850 a.C. (Almagro Gorbea, 1977: 525), el 900 a.C. (Ruiz-Gálvez, 1984: 283 y 1986: 11) o ya con fechas calibradas $c a$. 1000-700 AC (Ruiz-Gálvez, 1995: 82, 155).

Un fenómeno similar ha ocurrido con el depósito de Monte Sa Idda que también ha sido considerado tardío, sobre el 750 a.C. (Coffyn, 1985: 158) o 725-650 a.C. (Ruiz-Gálvez, 1986: 33), al tomarse uno de los artefactos más modernos del mismo para datarlo, caso de las espadas tipo $\mathrm{Sa}$ Idda. Sin embargo, Lo Schiavo (1991: 220) ha planteado que esta fecha sólo debe interpretarse como un terminus ante quem, que no necesariamente data todo el depósito, que a su juicio se encuadraría mayoritariamente entre el 1000800 a.C.

Una revisión de esta premisa es fundamental para interpretar la cronología de algunos depósitos italianos, sicilianos y especialmente sardos. La tesis partidaria de fecharlos de acuerdo con los artefactos más recientes es coherente porque se presupone la reutilización de los mismos. Sin embargo, cuando tratamos sobre posibles importaciones hay dos posibilidades que debemos barajar:

A) Los artefactos llegaron en cargamentos de chatarra de bronce en un momento relativamente próximo al de la realización del depósito. La interpretación del hallazgo de la ría de Huelva como el cargamento de un barco hundido con piezas para fundir (Almagro Basch, 1940: 85) reforzó esta línea interpretativa, pero no está claro que algunas roturas o dobleces de las piezas no hayan sido rituales, que parte del depósito no sea estrictamente sincrónico ya que aparece una espada RixheimMonza (Almagro Basch, 1958: lám. 11/51; Harrison, 1974-75: 230; Mederos, 1996, 97), o que realmente nos encontremos ante la asociación de un pecio y diversos depósitos rituales.

La idea de un comercio fenicio de chatarra hacia Cerdeña ha continuado siendo defendida por López Palomo (1978: 233), González Prats (1985: 97, 105) y particularmente Ruiz-Gálvez (1986: 34), quien fecha los depósitos de Sa Idda, Forraxi Nioi y Monte Arribu hacia el 650 a.C.

B) Las importaciones se produjeron en el pasado y son objeto de reutilización al descubrirse accidentalmente durante las reocupaciones de poblados, construcción de cimientos en Nuraghes, etc. Aquí, lo importante, para nuestros fines, no es la reutilización en sí, sino que la pieza debió haber arribado a la isla en un momento relativamente coetáneo a la cronología del artefacto. El escaso número y peso en gramos de estas piezas, más la abundancia de recursos cupríferos en la isla de Cerdeña, nos hace dudar de la llegada de barcos desde la Península Ibérica reciclando piezas, cuando habría otros artefactos más acordes con las modas y avances tecnológicos de cada momento, que sí serían objeto de mayor demanda. Un ejemplo clásico para España es la presencia de una punta de Palmela en el depósito del Bronce Final de Padilla de Abajo (Burgos) (MacWhite, 1951: 89 , lám. 22) junto a un hacha de talón con 2 anillas laterales y una punta de lanza con enmange tubular, que señalan claramente su reutilización tras ser recuperada presumiblemente en un contexto relativamente próximo al lugar del depósito.

Además, la revisión que ofrecemos de la cronología y distribución de los artefactos atlánticos en el Mediterráneo Occidental y Central revela un proceso continuado de intercambios entre la Península Ibérica, Cerdeña, Sicilia y Península meridional italiana a lo largo del Bronce Final.

Los artefactos documentados en el Mediterráneo Central pueden dividirse en tres grandes grupos: espadas, hachas y uno tercero heterogéneo, con un escaso número de presencias, que incluye puñales, puntas de lanza, cinceles, navajas, etc.

T. P., 54, n. $^{\circ} 2,1997$ 
Nuestro objetivo serán los dos primeros grupos, por su mayor abundancia y presumible procedencia de la Península Ibérica.

En segundo lugar se analizará el papel que jugó el Mediterráneo Central como región de interrelación entre las redes comerciales micénicas del Mediterráneo Oriental con las procedentes de la Península Ibérica. Dentro de esta nueva propuesta interpretativa se expone un modelo histórico explicativo frente al tratamiento de este problema a modo de fenómenos aislados, exóticos o discutibles que se otorga a la existencia de productos metalúrgicos atlánticos en Cerdeña, Sicilia e Italia continental, o a la cerámica micénica en Andalucía. Nuestro objetivo último será tratar de superar las lecturas del proceso histórico Mediterráneo durante el Bronce Final en forma de compartimentos estancos entre el Mediterráneo Occidental y el Oriental.

En tercer lugar, proponemos la correlación por primera vez entre dos fenómenos que siempre se han valorado de forma independiente, el comercio micénico que ha presentado en los últimos 15 años importantes novedades como la documentación de cerámica micénica en Cerdeña (Ferrarese Ceruti, 1979) y Andalucía (Martín de la Cruz, 1987) y el comercio atlántico con el Mediterráneo Central. Este último, tradicionalmente, se ha situado en fechas muy recientes, a partir del 1000 a.C. hasta su apogeo en el 800-700 a.C. para Coffyn (1985: 158-159, 274) y Ruiz-Gálvez (1986: 11, 17, 33 y 1993: 64). En este comercio, según alguna autora, intervendrían los fenicios al menos desde el 725 a.C. (Ruiz-Gálvez, 1986: 20, 34) y previamente, presuntamente, navegantes sardos (Ruiz-Gálvez, 1993: 62 y 1995: 145, 147), los cuales supuestamente llegaron a disponer de bases de operaciones en el centro de Portugal (Ruiz-Gálvez, 1995: 145), hipótesis aún por contrastar adecuadamente.

En conclusión, se trata de ofrecer una nueva lectura de los intercambios comerciales entre el Mediterráneo Central y Occidental a partir de tres premisas, la revisión de la cronología calibrada del Bronce Final en la Península Ibérica (Mederos 1995a: 86 y 1996: 98, tabla 2) y su articulación con las secuencias cronológicas calibradas del Mediterráneo Central y Oriental (Manning, 1995), que presentaremos siempre AC frente a las cronologias convencionales a.C. Se vuelve a evaluar la cronología de los artefactos atlánticos presentes en depósitos italianos a partir de su contrastación con las series ibéricas. Y, por último, se analizan en conjunto dos fenómenos históricos, la confluencia del comercio atlántico y el comercio micénico en el Mediterráneo Central. El objetivo final será continuar el análisis de las redes comerciales de la Península Ibérica durante la Prehistoria Reciente, concretamente entre el 1425-1050 AC, Bronce Final IC-IIC, siguiendo las premisas de los Sistemas Mundiales Antiguos (Mederos, 1995 b y 1996).

\section{LA RUTA OCCIDENTAL. PRODUCTOS ATLÁNTICOS IMPORTADOS O IMITADOS EN EL MEDITERRÁNEO CENTRAL}

Las hachas son los artefactos de producción o tradición del Bronce Final atlántico con una distribución más abundante en el Mediteráneo Central, $\mathrm{y}$ aunque siempre conviene un punto de prudencia, a falta de analíticas adecuadas, como mínimo responden a modelos atlánticos y, específicamente, ibéricos.

Aun sabiendo que habitualmente suelen observarse matices diferenciales en las hachas, lo que hace presuponer centros de producción relativamente locales, caso de la Península Ibérica, no hace falta más que echar un vistazo al catálogo de Italia continental (Carancini, 1984) para apreciar que entre una muestra de 4.487, sólo existen dos hachas planas de apéndices laterales en el depósito de Monte Rovello y una hacha de talón con una anilla.

\section{Hachas de apéndices laterales. Bronce Final IC-II}

La funcionalidad, origen y cronología de estas hachas siempre ha resultado muy polémica, y merece un tratamiento más detallado. Respecto a su funcionalidad, no faltan autores que defienden su uso como azuelas (Deshayes, 1960: 128; Almagro Gorbea, 1996: 275), escoplos (MacWhite, 1951: 55-56; Branigan, 1974: 23), lingotes de cobre (González Prats, 1985: 98), uso monetario (Siret, 1913: 364), e incluso cierto valor religioso por la representación de brazos rudimentarios a modo de ídolos-hachas antropomorfizados (Siret, 1913: 365-367).

Sobre su origen, la tesis mayoritaria ha defendido hasta la actualidad un precedente caucásico (Esparza, 1978: 348) o anatólico, que desde el Egeo, básicamente Troya y Grecia (Bosch Gimpe- 
ra, 1945: 107; Maxwell-Hyslop, 1953: 79; Almagro Basch, 1954: 27; Deshayes, 1960: 122; Harding, 1975: 186; Molina González, 1978: 215; Fernández Manzano, 1986: 75; Delibes y Fernández-Miranda, 1988: 118; Albanese, 1989: 188; Giardino, 1995: 200) se habría difundido hacia el Mediterráneo Occidental a través de Sicilia y Cerdeña, aunque no faltan tesis partidarias de un origen centroeuropeo (Fernández Gómez, 1974: 68). La presencia de un molde de hacha de apéndices laterales en la ciudad VII de Troya (Dörpfeld, 1902: 405, fig. 405), actualmente expuesto en el Museo de Estambul, fue el argumento básico esgrimido. El hallazgo estratificado de otra hacha de apéndices laterales en niveles del Heládico Final IIIC de Asino (Frödin y Persson, 1938: 311, fig. 214/2) reforzó esta supuesta primacía oriental.

Una alternativa diferente ha sido propuesta por Almagro Gorbea (1996: 274) quien atribuye las hachas de apéndices laterales italianas a una procedencia egea, mientras habría existido una introducción temprana directa en la Península Ibérica desde Siria y Palestina. Por nuestra parte, al igual que Lo Schiavo (1989: 110-120 y 1991: 215-216) y Albanese (1989: 193), nos inclinamos por un origen ibérico para los ejemplares de Cerdeña, Sicilia y Península Italiana, sin descartar alguna producción local tardía en Sicilia, como también se realizaron en Grecia y Anatolia.

La tesis de una vía griega para los ejemplares italianos tiene tres inconvenientes. En principio, está el problema de su escasa representación en el Egeo, apenas 7 ejemplares: Grecia (Asino, Anthedon, Araxos, Teichos Dymaion), Rodas (Lindos), Cos (Seraglio) y Creta (Karphi), aunque existen también dos moldes, Assiros Toumba (Grecia) y Troya, no dudando Wardle (1989: 458) en considerarlo un tipo inusual.

En segundo lugar, está el carácter más tardío de estos ejemplares, generalmente de excavaciones no bien contextualizadas, puesto que el hacha de Araxos (Harding, 1975: 184) procede de un nivel mezclado de Heládico Inicial con presumible Heládico Final IIIC, y el de Teichos Dymaion (Dakaris, 1985: 112-113, fig. 2a) oscila entre el Heládico Final IIIB o IIIC. El molde de Assiros Toumba, en Macedonia (Wardle, 1989: 458, fig. 3 ), se correlaciona con un momento avanzado del Heládico Final IIIC2. Los procedentes de Anthedon, Lindos y Seraglio (Blinkenberg, 1931: 68, lám. 3/27; Buchholz y Karageorghis, 1971: ${ }^{\circ}$ 554) son genéricamente Heládico Final III. El de la ciudad VII de Troya se correlaciona con el Heládico Final IIIC y el Protogeométrico Griego, 1250-1000 AC. El de Karphi (Pendlebury et alii, 1937-38: 88, 116, lám. 29/2/455) corresponde al Protogeométrico, en contraposición a la cronología que proponemos para el tipo 20A, Bronce Final IC-II, $c a$. 1425-1150 AC.

Finalmente, existen divergencias tipológicas entre los ejemplares egeos y las hachas italianas e ibéricas con apéndices laterales menos marcados o más anchos, y talón más desarrollado longitudinalmente, siendo el mejor paralelo con los ibéricos el molde de Troya, mientras el ejemplar de Assiros presenta apéndices laterales muy anchos.

Las hachas de apéndices laterales del tipo 20A, asignables al Bronce Final IC-II ( Fig. 1; Tab. 1), se concentran particularmente en el Norte de la provincia de Teruel, y se distribuyen exclusivamente en la vertiente mediterránea, Cuenca, Jaén y, ya próximas a la costa, Alicante y Murcia. Aunque sería deseable disponer de buenas secciones para algunos ejemplares de Cerdeña, de algunos casos (Lo Schiavo, 1980: abb. 103) parece inferirse la asignación al tipo 20A. No obstante, una modificación del mismo implicaría distribuciones más orientadas hacia el centro de Portugal y Cáceres.

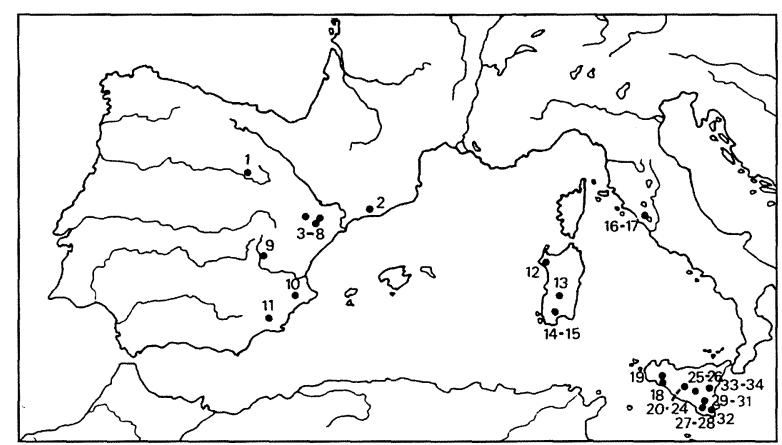

Fig. 1. Distribución de las hachas de apéndices laterales en la Península Ibérica, Cerdeña y Sicilia. Tipo 20A. 1: Cuesta del Moro (Soria). 2: Barcelona. 3: El Castelillo (Teruel). 4: Ejulve (Teruel). 5: Iglesuela del Cid (Teruel). 6: La Iglesia (Teruel). 7-8: Teruel. 9: Alarcón (Cuenca). 10: La Serreta (Alicante). 11: Monte de Santa Catalina (Murcia). 12: Nuraghe Flumenelongu (Sassari). 13: Albini (Nuoro). 14-15: Monte Sa Idda (Cagliari). 16-17: Monte Rovello (Roma). 18: Agriento. 19: Polizello (Caltanissetta). 20-24: Niscemi (Gela). 25: Piazza Armerina (Enna). 26: Enna. 27-28: Modica (Ragusa). 29-31: Castelluccio (Ragusa). 32: Noto Antica (Siracusa). 33: Paterno (Catania). 34: Catania. 


\begin{tabular}{|c|c|c|c|c|c|c|}
\hline Yacimiento & $\begin{array}{l}\text { Municipio- } \\
\text { comune }\end{array}$ & Provincia & Región & Tipo & Clase & Bibliografía \\
\hline \multirow[t]{2}{*}{ Cuesta del Moro } & Langa de Duero & Soria & Castilla-León & poblado & $20 \mathrm{~A}$ & Monteagudo, 1977: 139, 828 \\
\hline & & Barcelona & Cataluña & & $20 \mathrm{~A}$ & Monteagudo, 1977: 139, 820A \\
\hline \multirow{3}{*}{ El Castelillo } & Alloza & Teruel & Aragón & & $20 \mathrm{~A}$ & Monteagudo, 1977: 139, 821 \\
\hline & Ejulve & Teruel & Aragón & & $20 \mathrm{~A}$ & Monteagudo, 1977: 139, 822 \\
\hline & Iglesuela del Cid & Teruel & Aragón & & $20 \mathrm{~A}$ & Monteagudo, 1977: 139, 823 \\
\hline \multirow[t]{3}{*}{ La Iglesia } & & Teruel & Aragón & & $20 \mathrm{~A}$ & Monteagudo, 1977: 139, 824 \\
\hline & & Teruel & Aragón & & $20 \mathrm{~A}$ & Monteagudo, 1977: 139, 825 \\
\hline & & Teruel & Aragón & & $20 \mathrm{~A}$ & Monteagudo, 1977: 139, 829 \\
\hline $\begin{array}{l}\text { Oeste de Montilla } \\
\text { del Palancar }\end{array}$ & Alarcón & Cuenca & Castilla-La Mancha & & $20 \mathrm{~A}$ & Monteagudo, 1977: 139, 826 \\
\hline \multirow{2}{*}{$\begin{array}{l}\text { La Serreta } \\
\text { Monte de Santa } \\
\text { Catalina }\end{array}$} & Alcoy & Alicante & País Valenciano & poblado & $20 \mathrm{~A} / \mathrm{B}$ & Simón, 1995: 178-179, fig. 2a \\
\hline & Verdolay, Murcia & Murcia & Murcia & poblado & $20 \mathrm{~A}$ & Monteagudo, 1977: 139, 827 \\
\hline \multicolumn{6}{|l|}{ Nuraghe } & Lo Schiavo, 1976: 10, tav. VI/1 \\
\hline Abini & Teti & Nuoro & Cerdeña & santuario & $20 \mathrm{~A}$ & $\begin{array}{l}\text { Pinza, 1901: 170, fig. 90; Monteagudo, } \\
\text { 1977: } 140\end{array}$ \\
\hline Monte Sa Idda & Decimoputzu & Cagliari & Cerdeña & depósito & $20 \mathrm{~A}$ & $\begin{array}{l}\text { Taramelli, 1921: 19, fig. 12; Monteagudo, } \\
\text { 1977: } 140\end{array}$ \\
\hline \multirow[t]{2}{*}{ Monte Sa Idda } & Decimoputzu & Cagliari & Cerdeña & depósito & 20 & Taramelli, 1915: 92, fig. 2 \\
\hline & & & Cerdeña & & 20 & $\begin{array}{l}\text { Pinza, 1901: tav. XVII/16; Lo Schiavo, } \\
\text { 1991: } 216\end{array}$ \\
\hline \multirow[t]{2}{*}{ Monte Rovello (2) } & Allumiere & Roma & Lazio & depósito & $20 \mathrm{~A}$ & $\begin{array}{l}\text { Montelius, 1895: pl. 121/26; Peroni, } \\
\text { 1961: 1.2.4/4; Carancini, 1984: 235, } \\
\text { tav. 172/4486-4487 }\end{array}$ \\
\hline & & Agriento & Sicilia & & & Giardino, 1995: 202 fig. 93/2, 205 \\
\hline Polizzello & Mussomeli & Caltanissetta & Sicilia & & & Giardino, 1995: 202 fig. $93 / 6,205$ \\
\hline $\begin{array}{l}\text { Niscemi (4) } \\
\text { (1 atípico) }\end{array}$ & Gela & Caltanissetta & Sicilia & depósito & $20 \mathrm{~A}$ & $\begin{array}{l}\text { Müller-Karpe, 1959: taf. 12/C3; Albanese, } \\
\text { 1989: 195-196, 200-201 fig. 3-7 }\end{array}$ \\
\hline \multirow[t]{3}{*}{ Piazza Armerina } & & Enna & Sicilia & & & $\begin{array}{l}\text { Orsi, 1898: 163, fig. 2; Albanese, 1989: } \\
\text { 195, } 200 \text { fig. 2. Apéndices no simétricos }\end{array}$ \\
\hline & & Enna & Sicilia & & 20 & Giardino, 1995: 202 fig. $93 / 7,205$ \\
\hline & Modica (2) & Ragusa & Sicilia & depósito & 20 & Orsi, 1900: 167 , tav. XII/14,18 \\
\hline \multirow[t]{4}{*}{ Castelluccio (3) } & & Ragusa & Sicilia & depósito & 20 & $\begin{array}{l}\text { Di Stefano, 1984; di Stefano y Giardino, } \\
\text { 1990-91: } 499 \text {, fig. } 6 / 2-4,503\end{array}$ \\
\hline & Noto Antica & Siracusa & Sicilia & depósito & 20 & $\begin{array}{l}\text { Albanese, } 1986-87: 46 \text {, tav I/1 y } 1989 \text { : } \\
\text { 198, } 201 \text { fig. } 8\end{array}$ \\
\hline & Paterno & Catania & Sicilia & & 20 & $\begin{array}{l}\text { Albanese, } 1986-87: 49, \text { tav. I/6 y } 1989: \\
195,200 \text { fig. } 1\end{array}$ \\
\hline & & Catania & Sicilia & & 20 & Giardino, 1995: 202 fig. $93 / 5,205$ \\
\hline
\end{tabular}

Tab. 1. Distribución de las hachas de apéndices laterales en la Península Ibérica, Cerdeña y Sicilia. Tipo 20A. El hacha de Pozzuoli (Campania) (Mayron, 1938: 248-249, fig. 19) era demasiado ancha y tenía los apéndices laterales muy bajos. En su reciente revisión en el British Museum por Giardino (1995: 200), lo ha identificado como un hacha de márgenes resaltados del Bronce Inicial.

En Cerdeña, se concentran en la mitad S.SO. y N.NO., incluyendo el Campidano, la región agrícola más rica de la isla, o las comarcas de Marmilla y Logudoro, ambas también con buen aprovechamiento agrícola. Ello contrasta completamente con el patrón de las espadas pistiliformes del Bronce Final II (vide infra, Fig. 3), orientado hacia las comarcas del Noreste y centro orientales.

La cronología de la hachas de apéndices siempre ha resultado problemática, pero está también sujeta al subtipo del que estamos tratando, lo que no siempre se especifica. Para el tipo 19A de Monteagudo (1977), Almagro Gorbea (1996: 275), basándose en el depósito de Osuna con un hacha de talón y una anilla, propone un Bronce Final I coetáneo a Rosnöen, 1200-1000 a.C., que nosotros fechamos en el Bronce Final IC, ca. 1425-1325 AC.

Para el tipo 20A1, Fernández Manzano (1986: 78), Delibes y Fernández-Miranda (1988: 119) proponen un Bronce Final II, apoyándose en el 
depósito de Covaleda (Soria), con 2 hachas de talón y una anilla, una hacha de talón y dos anillas y un regatón de lanza.

El tipo 19B, representado sólo por el ejemplar de La Sabina (Formentera), asociado a un hacha de cubo, Delibes y Fernández Miranda (1988: 119) lo sitúan entre el 800-700 a.C. y Fernández Gómez (1973: 183) entre el 800-600 a.C. Los tipos 19A, 20A, 20B y 20C, Ruiz-Gálvez (1986: $14,17)$ los encuadra hacia el 800-700 A.C.

Finalmente, para el tipo 22A, Delibes y Fernández-Miranda (1988: 119) y Fernández-Miranda (1993: 149, 156) apuntan el 800-700 a.C., Fernández Gómez (1974: 70) hacia el 750-500 a.C., y González Prats (1985: 98), a partir de hachas estratificadas en el poblado de Peña Negra, entre el 700-550 a.C.

Sin embargo, para el tipo 20A no contamos con datos indicativos precisos por falta de contextos o asociaciones adecuadas, y sí con dos reutilizaciones, una en el poblado celtibérico de Langa de Duero (Soria), junto a cinco denarios ibéricos, y otra en el departamento 5, sector G, del poblado ibérico de La Serreta (Alicante), asignable a los siglos IV-III a.C. (Simón, 1995: 178, 181). No obstante, la pieza más importante es el molde de fundición de hachas de apéndices del poblado del Cerro de Santa Catalina del Monte en Verdolay (Murcia) (Ros, 1986: 333, fot. 1) que implica la fabricación in situ de este tipo en la Península Ibérica, y específicamente en el Sureste, a pesar de su mayoritaria distribución en Teruel, de donde proceden 6 de los 10 ejemplares de la Península Ibéri- ca. De este poblado contamos con una fuente con carena alta y decoración de boquique con motivos de guirnalda (Fernández Avilés, 1935: fig. 1) asignable al Bronce Final I-II, puesto que en la fase 16 de Fuente Álamo (Almería) disponemos de cerámicas decoradas tipo Cogotas al menos desde el 1525 AC (Schubart y Arteaga, 1983; Mederos, 1995a: 69), en el Bronce Final IB.

Estos datos nos sugieren al menos una cronología del Bronce Final IC para el tipo 20A de las hachas de apéndices laterales. Las coincidencias en la distribución mediterránea, particularmente centrada en el valle del Ebro, también presente en el tipo 20A1, que se dispersa entre Navarra, Soria y Zaragoza, ayudan a sostener la continuidad de este artefacto durante el Bronce Final II, tal como apunta el depósito de Covaleda (Boca del Prado, Soria) (Ortego, 1957: 116-117).

\section{Hachas de talón de sección hexagonal y dos anillas. Bronce Final IC-II}

Desde un momento del Bronce Final IC y, claramente ya durante el Bronce Final II, el hacha de tipo $26 \mathrm{C} 2$ se concentra alrededor de dos cuencas fluviales, la vertiente septentrional del río Duero, con piezas en León, Palencia y Burgos, y simultáneamente, la cuenca alta del río Ebro, con hachas en el Norte de Burgos y Navarra. El hecho de que el río Ebro fuese navegable en época romana hasta Logroño implica una vía alternativa hacia el Mediterráneo sin necesidad de seguir el río Duero hasta el Atlántico (Tab. 2; Fig. 2).

\begin{tabular}{|l|l|l|l|c|l|}
\hline \multicolumn{1}{|c|}{ Yacimiento } & \multicolumn{1}{|c|}{ Municipio-comune } & \multicolumn{1}{|c|}{ Provincia } & \multicolumn{1}{c|}{ Región } & \multicolumn{1}{c|}{ Tipo } & \multicolumn{1}{c|}{ Bibliografía } \\
\hline & Reliegos & León & Castilla-León & & Celis, 1985 inédito en Delibes et alii, 1994: 261 \\
\hline ¿Saldaña? & Dehesa de Romanos & Palencia & Castilla-León & & $\begin{array}{l}\text { Monteagudo, 1977: 156, 931; Delibes } \text { et alii, } \\
\text { 1994: 250 }\end{array}$ \\
\hline Pico Cuerno & Sotoscueva & Burgos & Castilla-León & depósito & $\begin{array}{l}\text { Monteagudo, 1977: 158, 937; Delibes } \text { et alii, } \\
\text { 1994: 246, fig. 2/1 }\end{array}$ \\
\hline ¿Castro? & Renedo de Amaya & Burgos & Castilla-León & & Monteagudo, 1977: 158, 938 \\
\hline & Valdelateja & Burgos & Castilla-León & & Monteagudo, 1977: 158, 940 \\
\hline & Quintana de Bureba & Burgos & Castilla-León & & $\begin{array}{l}\text { Monteagudo, 1977: 156, 931; Delibes } \text { et alii, } \\
\text { 1994: 250 }\end{array}$ \\
\hline & Padilla de Abajo & Burgos & Castilla-León & depósito & Monteagudo, 1977: 158, 939 \\
\hline & & Burgos & Castilla-León & & Monteagudo, 1977: 158, 941 \\
\hline Peña del Saco & Fitero & Navarra & Navarra & poblado & Monteagudo, 1977: 158, 942 \\
\hline Nuraghe Flumenelongu & Alghero & Sassari & Cerdeña & depósito & Lo Schiavo, 1976: 9-10, tav. III-IV \\
\hline Monte Sa Idda (3) & Decimoputzu & Cagliari & Cerdeña & depósito & $\begin{array}{l}\text { Taramelli, 1921:21, fig. 16-18; Monteagudo, } \\
1977: 159\end{array}$ \\
\hline Monte Arrubiu & Sarroch & Cagliari & Cerdeña & depósito & $\begin{array}{l}\text { Taramelli, 1926: 447, fig. 17; Lo Schiavo, 1990: } \\
\text { 254, fig. 249/3; Monteagudo, 1977: 159 }\end{array}$ \\
\hline
\end{tabular}

Tab. 2. Distribución de las hachas de talón con sección hexagonal y dos anillas en la Península Ibérica y Cerdeña. Tipo 26C2.

T. P., 54, n. ${ }^{\circ} 2,1997$ 


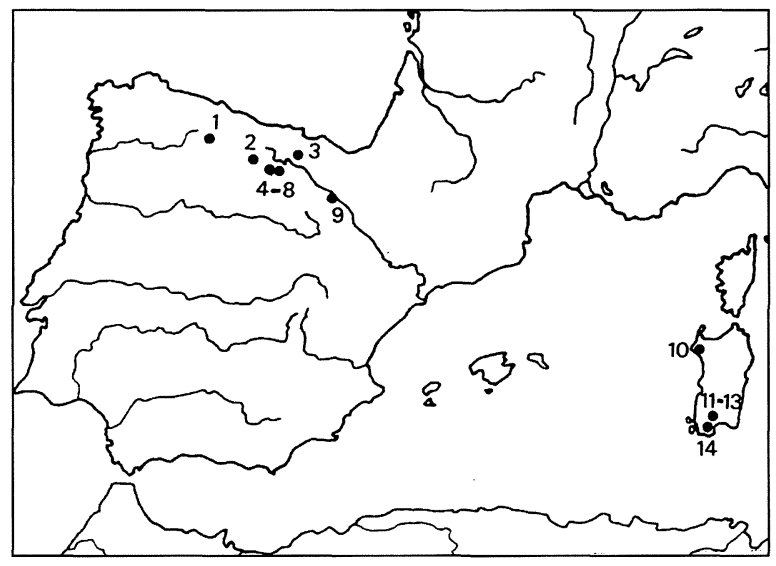

Fig. 2. Distribución de las hachas de talón con sección hexagonal y dos anillas en la Península Ibérica y Cerdeña. Tipo 26C2. 1: Reliegos (León). 2: Dehesa de Romanos (Palencia). 3: Pico Cuernos (Burgos). 4: Renedo de Amaya (Burgos). 5.Valdelateja (Burgos). 6: Quintana de Bureba (Burgos). 7: Padilla de Abajo (Burgos). 8: Burgos. 9: Peña del Saco (Navarra). 10: Nuraghe Flumenelongu (Sassari). 11-13: Monte Sa Idda (Cagliari). 14: Monte Arrubiu (Cagliari).

Las tres hachas documentadas en Cerdeña se localizan junto a dos de los mejores puertos de la isla, Nuraghe Flumenelongu próximo a Porto Conte en la comarca de La Nurra del N.NO., mientras Sa Idda y Monte Arrubi enmarcan el golfo y puerto meridional de Cagliari. Si bien Delibes et alii (1994: 265-266) propugnan simultáneas producciones independientes en la Península Ibérica y Cerdeña, en todo caso imitarían producciones específicamente ibéricas.

\section{Espadas pistiliformes. Bronce Final II}

Actualmente se conocen cinco espadas de este tipo, dos de ellas procedentes de Su Tempiesu, conservándose completa sólo la espada de Oroè (Pesce, 1952: fig. 44). Existe, además, la referencia, nunca demostrada, de otros cinco ejemplares procedentes de Ozieri (Zervos, 1954: 195). A. Coffyn (1985: 149) valora la espada de Oroè como una posible derivación local de modelos portugueses, sin embargo, Lo Schiavo (1991: 219) la considera el ejemplar mas claro de espada pistiliforme importada, optando por asignar a las demás espadas una hipotética fabricación local.

Si nos remitimos a la Península Ibérica, la distribución de las mismas (Fig. 3) resulta bastante homogénea en todo el territorio ibérico, quizás

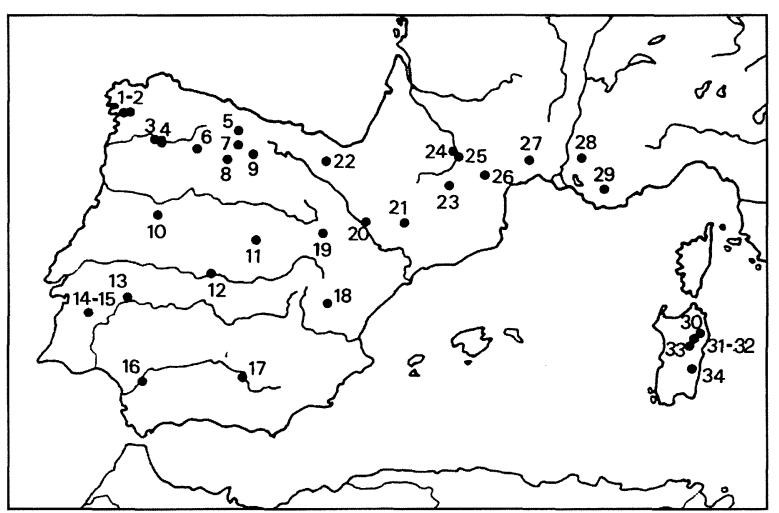

Fig. 3. Distribución de las espadas pistiliformes en la Península Ibérica, S. de Francia y Cerdeña. 1-2: Río Ulla (Pontevedra); 3: San Esteban del Río Sil (Orense). 4: Mouruás (Orense); 5. Sobrefoz (Asturias). 6: La Cabrera-Bierzo (León). 7: Río Esla (León). 8: Veguellina de Orbigo (León). 9: Villaverde de la Chiquita (Palencia). 10: Vilar Maior (Beira Alta). 11: Segovia. 12: Vado de AzutánCarpio del Tajo (Toledo). 13: Montijo (Badajoz). 14-15: Alemtejo-Evora (Alto Alemtejo). 16: Dos Hermanas-Corta de la Cartuja (Sevilla). 17: Vado de Menjíbar (Jaén). 18: Carboneras de Guadazaón (Cuenca). 19: Alhama de Aragón (Zaragoza). 20: Zaragoza. 21: Binefar (Huesca). 22: Solacueva de Lacozmonte (Álava). 23: Le Masd'Azil (Ariège). 24: Le Bazacle (Haute-Garonne). 25: Clermont-le-Fort (Haute-Garonne). 26: Trebes (Aude). 27: Patus de la Vacquière (Hérault). 28: Malaucène (Vaucluse). 29: La Farigourière (Var). 30: Oroè (Nuoro). 31-32: Su Tempiesu (Nuoro). 33: Bolòtana (Nuoro). 34: Ogliastra (Nuoro).

\begin{tabular}{|l|l|l|l|l|}
\hline \multicolumn{1}{|c|}{ Yacimiento } & \multicolumn{1}{c|}{ Municipio-comune } & \multicolumn{1}{c|}{ Provincia } & \multicolumn{1}{c|}{ Tipo } & \multicolumn{1}{c|}{ Bibliografía } \\
\hline Oroè & Siniscola & Nuoro & depósito & Pesce, 1952: 187-188, fig. 44 \\
\hline Su Tempiesu & Orune & Nuoro & santuario & Fadda, 1988: 23; Lo Schiavo, 1991: 219, fig. 4/2 y 3 \\
\hline Bolòtana & Bolòtana & Nuoro & santuario & Taramelli, 1911: 312, nota 2; Lo Schiavo, 1991: 219, fig. 4/1 \\
\hline & Ogliastra & Nuoro & ¿hallazgo aislado? & Pinza, 1901: 212 \\
\hline
\end{tabular}

Tab. 3. Espadas pistiliformes en Cerdeña. 
con una cierta concentración en la provincia de León y en el Valle Medio del río Duero. En este sentido, resulta llamativa su distribución al interior de la Península, sin la distribución marcadamente costera en áreas como Bretaña. Esta concentración en las comarcas interiores se repite en la distribución de las espadas en Cerdeña (Tab. 3; Fig. 3), que además se agrupan en el cuadrante Noreste de la isla, aproximadamente entre Ogliastra, Nuorese y Baronie, regiones donde el poblamiento nurágico no resulta especialmente denso, con suelos más pobres excepto alrededor de algunos valles costeros, escasos recursos metalúrgicos y presumible mayor orientación pastoril. Creemos interesante plantear la posibilidad de que algunos miembros de las élites propietarias de ganado de esas regiones fuesen quienes más demandasen este tipo de armamento de prestigio.

La ausencia de espadas en el País Valenciano y Cataluña apoyaría la llegada a Cerdeña de algún ejemplar, o al menos de la moda de su uso, bien desde Andalucía Occidental o el Valle del Ebro, bien desde el litoral atlántico ibérico. No obstante, si inicialmente Guilaine (1972: 235-236, 237 fig. 81/1-2) señalaba que los ejemplares franceses más meridionales correspondían a las espadas de $\mathrm{Ba}$ - zacle en el Alto Garona, y de Lasbordes en Tarn, ambas en la región de Midi-Pyrénées, los datos más recientes (Guilaine et alii, 1991: 308-310) han ampliado su distribución al Languedoc y la Provenza.

\section{Hachas de talón con una anilla. Bronce Final II}

El hacha tipo 34A, tiene su núcleo en el Norte de Portugal, Minho, Tras-os-Montes, Beira Alta, con límites en La Coruña, Santander, Norte de Cáceres y Beira Baja, salvo dos extensiones puntuales hacia Guadalajara y la Península de Lisboa (Tabla 4; Fig. 4).

Un dato que llama la atención es su ausencia de Cerdeña, y presencia por el contrario en la Italia continental. Sin embargo, Carancini (1984: 142) apunta un origen sardo y, genéricamente, del Mediterráneo Occidental, para el ejemplar de Tolfa. Simultáneamente, las hachas del depósito siciliano de Castelluccio indican la continuidad de la ruta abierta desde el Bronce Final IC que ya apuntaban las hachas de apéndices laterales presentes en Sicilia.

\begin{tabular}{|c|c|c|c|c|c|}
\hline Yacimiento & Municipio-comune & Provincia & Región & Tipo & Bibliografía \\
\hline \multirow[t]{2}{*}{ Muiño da Granxa } & Senra & La Coruña & Galicia & depósito & Monteagudo, 1977: 205, 1301 \\
\hline & Landrove & Lugo & Galicia & & Monteagudo, $1977: 205,1301 \mathrm{~A}$ \\
\hline \multirow[t]{2}{*}{ Rasa de Luces } & Lastres & Asturias & Asturias & depósito & Monteagudo, 1977: 205, 1302 \\
\hline & Requejo & Santander & Santander & & Monteagudo, $1977: 205,1303$ \\
\hline \multirow[t]{2}{*}{ Vidual } & Linhares, Villa Real & Tras-os-Montes & Norte & & Monteagudo, 1977:204, 1298 \\
\hline & Chaves & Tras-os-Montes & Norte & & Monteagudo, 1977: 204, 1299 \\
\hline \multirow[t]{5}{*}{ Cimadevlia } & $\begin{array}{l}\text { São Paio de Portela, } \\
\text { Penafiel }\end{array}$ & Douro Litoral & Norte & & Monteagudo, 1977:204, 1300 \\
\hline & Penafiel & Douro Litoral & Norte & & Monteagudo, $1977: 204-205,1300 \mathrm{~A}$ \\
\hline & Tourais, Seia & Beira Alta & Centro & ¿depósito? & Monteagudo, 1977: 204, 1295 \\
\hline & Viseu & Beira Alta & Centro & & Monteagudo, 1977:204, 1296 \\
\hline & Viseu & Beira Alta & Centro & & Monteagudo, 1977: 204, 1297 \\
\hline Monte do Crasto & $\begin{array}{l}\text { Vila Cova de Perrinho, } \\
\text { Vale de Cambra }\end{array}$ & Beira Litoral & Centro & $\begin{array}{l}\text { depósito en } \\
\text { poblado }\end{array}$ & Monteagudo, $1977: 204,1297 \mathrm{~A}$ \\
\hline \multirow[t]{2}{*}{ Ervedal (3) } & Castelo Novo & Beira Baixa & Centro & depósito & Monteagudo, 1977: 204, 1290-1292 \\
\hline & Vila Nova da Rainha & Ribatejo & Lisboa-Vale do Tejo & & Monteagudo, $1977: 204,1289$ \\
\hline \multirow[t]{3}{*}{ Descargamaría } & & Cáceres & Extremadura & & Monteagudo, 1977: 204, 1293 \\
\hline & & Cáceres & Extremadura & & Monteagudo, 1977: 204, 1294 \\
\hline & Fuente Sabiñán & Guadalajara & Castilla-La Mancha & & Monteagudo, $1977: 205,1303 \mathrm{~A}$ \\
\hline Massiccio della Tolfa & & Roma & Lazio & depósito & $\begin{array}{l}\text { Peroni, 1961: 1.3.5; Carancini, 1984: 142, tav, } \\
\text { 120/3715 }\end{array}$ \\
\hline \multirow[t]{2}{*}{ Castelluccio (2) } & & Ragusa & Sicilia & depósito & $\begin{array}{l}\text { Di Stefano y Giardino, 1990-91: } 502 \text { fig. 9/10-11, } \\
503\end{array}$ \\
\hline & & Siracusa & Sicilia & & Bernabó Brea, 1961: fig. $35 / 5$ \\
\hline
\end{tabular}

Tab. 4. Distribución de las hachas de talón con una anilla en la Península Ibérica, Italia y Sicilia. Tipo 34A.

T. P., 54, n. ${ }^{\circ} 2,1997$ 


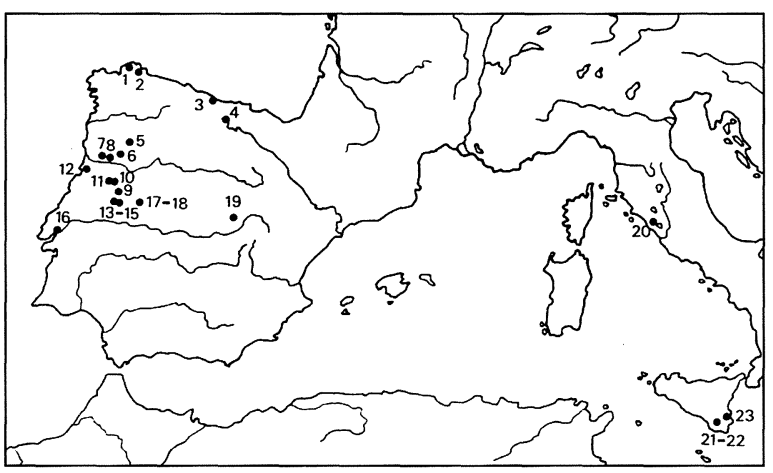

Fig. 4. Distribución de las hachas de talón con una anilla en la Península Ibérica, Italia y Sicilia. Tipo 34A. 1: Muiño da Granxa (La Coruña). 2: Landrove (Lugo). 3: Rasa de Luces (Asturias). 4: Requejo (Santander). 5: Vidual (Tras-os-Montes). 6: Chaves (Tras-os-Montes). 7: Cimadevlia (Douro Litoral). 8: Penafiel (Douro Litoral). 9: Tourais (Beira Alta). 10-11: Viseu (Beira Alta). 12: Monte do Crasto (Beira Litoral). 13-15: Ervedal (Beira Baixa). 16: Vila Nova da Rainha (Ribatejo). 17: Descargamaría (Cáceres). 18: Cáceres. 19: Fuente Sabiñán (Guadalajara). 20: Massiccio della Tolfa (Roma). 21-22: Castelluccio (Ragusa). 23: Siracusa.

\section{Hachas de talón con dos anillas y nervadura central. Bronce Final II}

Una mayoritaria distribución en el cuadrante Noroeste de la Península Ibérica continúa en el hacha tipo 35B, quizás aún más costreñida al litoral atlántico con sus límites septentrionales y

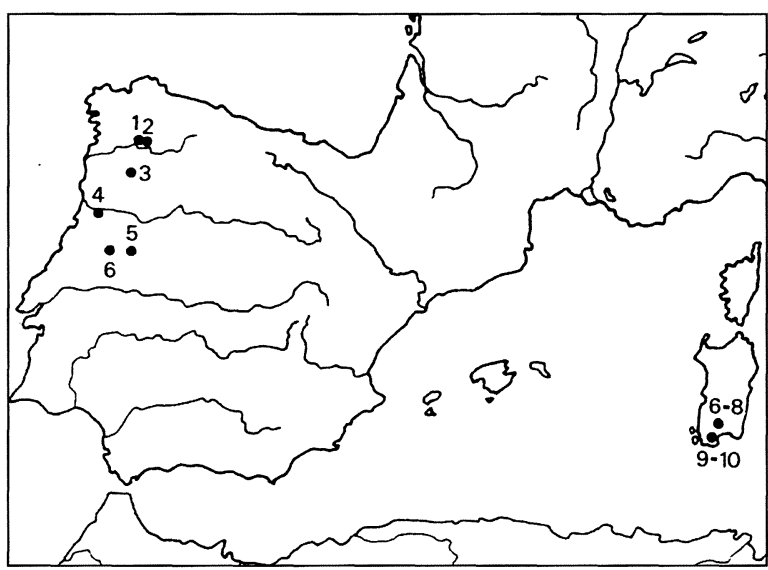

Fig. 5. Distribución de las hachas de talón con dos anillas y nervadura central en la Península Ibérica, Cerdeña y Sicilia. Tipo 35B. 1-2: Carballo (Lugo). 3: Tras-os-Montes. 4: Retorta (Douro Litoral). 5: Lajeosa de Raia (Beira Alta). 6: Paul (Beira Baixa). 6-8: Monte Sa Idda (Cagliari). 910: Monte Arrubiu (Cagliari). 11: Castelluccio (Ragusa).

meridionales entre Lugo y la Beira Baja (Tab. 5; Fig. 5).

En Cerdeña se concentran en el cuarto meridional de la isla, principalmente en torno al golfo de Cagliari, cuyo puerto es el mejor punto de arribada a la isla. Finalmente, el depósito de Castelluccio en la región siciliana de Ragusa nuevamente se correlaciona bien con una ruta marítima descendente desde el golfo de Cagliari por la vertiente costera meridional de Sicilia.

\begin{tabular}{|l|l|l|l|l|l|}
\hline \multicolumn{1}{|c|}{ Yacimiento } & \multicolumn{1}{|c|}{ Municipio-comune } & \multicolumn{1}{c|}{ Provincia } & \multicolumn{1}{c|}{ Región } & \multicolumn{1}{c|}{ Tipo } & \multicolumn{1}{c|}{ Bibliografia } \\
\hline Carballo (2) & $\begin{array}{l}\text { Hospital de Quiroga, } \\
\text { Quiroga }\end{array}$ & Lugo & Galicia & depósito & Monteagudo, 1977: 208, 1325-1326 \\
\hline & ¿Chaves? & Tras-os-Montes & Norte & & Monteagudo, 1977: 209, 1331 \\
\hline Retorta & Souzelo & Douro Litoral & Norte & idepósito? & Monteagudo, 1977: 209, 1329 \\
\hline & Lajeosa de Raia & Beira Alta & Centro & idepósito? & Monteagudo, 1977: 208-209, 1328 \\
\hline & & ¿Beira Alta? & Centro & & Monteagudo, 1977: 209, 1330 \\
\hline & Paul & Beira Baixa & Centro & & Monteagudo, 1977: 208, 1327 \\
\hline Monte Sa Idda (3) & Decimoputzu & Cagliari & Cerdeña & depósito & $\begin{array}{l}\text { Taramelli, 1921: 24, fig. 20, 23, 24; Monteagudo, } \\
\text { 1977: 209; Lo Schiavo, 1989: 111-112, fig. 3/7 }\end{array}$ \\
\hline Monte Arrubiu (2) & Sarroch & Cagliari & Cerdeña & depósito & $\begin{array}{l}\text { Taramelli, 1926: 447-448, fig. 18; Lo Schiavo, } \\
\text { 1990: 254, fig. 249/1-2 }\end{array}$ \\
\hline Castelluccio & & Ragusa & Sicilia & depósito & $\begin{array}{l}\text { Di Stefano, 1984; Di Stefano y Giardino, 1990-91: } \\
502 \text { fig. 9/12, 503 }\end{array}$ \\
\hline
\end{tabular}

Tab. 5. Distribución de las hachas de talón con dos anillas y nervadura central en la Península Ibérica, Cerdeña y Sicilia. Tipo 35B. 


\section{Hachas de talón unifacial con una anilla. Bronce Final IIC}

Sólo conocemos ejemplares para el Mediterráneo Central, en el depósito de Monte Sa Idda. La falta de buenas secciones nos impide pronunciarnos definitivamente entre el subtipo propuesto por Monteagudo (1977: 140), tipo 36B, que preferimos al del 36A de Ruiz-Gálvez (1986: 15). En todo caso las distribuciones de ambas son muy concretas dentro del litoral atlántico, el 36B, alrededor del curso bajo del río Tajo, Beira Litoral, Beira Alta y Extremadura portuguesa (Tab. 6; Fig. 6), y el 36A, en las Beiras Litoral, Alta y Baja.

Conviene recalcar que todas las hachas de talón siguen el patrón de distribución regional marcado desde las hachas de apéndices laterales, concentrándose en la mitad occidental de la isla de Cerdeña, y que se mantendrá igualmente $a$ posteriori con las hachas tubulares del Bronce Final IIIB. Ello sigue resultando manifiestamente contrapuesto a la distribución que presentan las espadas pistiliformes, todas en la mitad oriental.

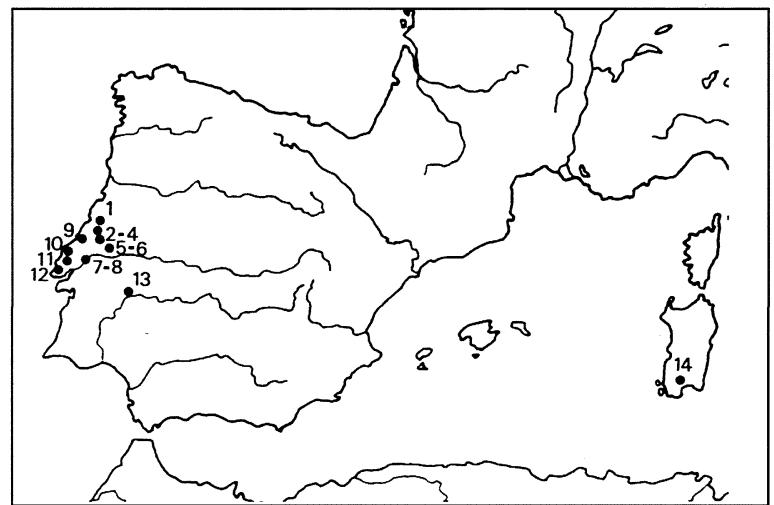

Fig. 6. Distribución de las hachas de talón unifacial con una anilla en la Península Ibérica y Cerdeña. Tipo 36B. 1: ¿Coimbra? (Beira Litoral). 2: Leiría-Alcobaça (Beira Litoral). 3: Serra de Alvaiazere (Beira Litoral). 4: Soure (Beira Litoral). 56: Pôrto do Concelho (Beira Baixa). 7-8: Monte de São Martinho (Ribatejo). 9: Castro de Pragança (Estremadura portuguesa). 10: Curral das Cabras (Estremadura portuguesa). 11: Cesareda (Estremadura portuguesa). 12: Cabeço de Moinhos (Estremadura portuguesa). 13: ¿Elvas? (Alto Alemtejo). 14: Monte Sa Idda (Cagliari).

\begin{tabular}{|l|l|l|l|l|l|}
\hline \multicolumn{1}{|c|}{ Yacimiento } & \multicolumn{1}{|c|}{ Municipio-comune } & \multicolumn{1}{c|}{ Provincia } & \multicolumn{1}{c|}{ Región } & \multicolumn{1}{c|}{ Tipo } & \multicolumn{1}{c|}{ Bibliografía } \\
\hline & ¿Coimbra? & Beira Litoral & Centro & & Monteagudo, 1977: 213, 1352 \\
\hline & Leiría-Alcobaça & Beira Litoral & Centro & depósito & Monteagudo, 1977: 212, 1349 \\
\hline Serra de Alvaiazere & Alvaiazere & Beira Litoral & Centro & & Monteagudo, 1977: 212-213, 1350 \\
\hline & Soure & Beira Litoral & Centro & depósito & Monteagudo, 1977: 213, 1351 \\
\hline $\begin{array}{l}\text { Pôrto do } \\
\text { Concelho (2) }\end{array}$ & Mação & Beira Baixa & Centro & depósito & Monteagudo, 1977: 212, 1347-1348 \\
\hline $\begin{array}{l}\text { Monte de São } \\
\text { Martinho (2) }\end{array}$ & Teira & Ribatejo & $\begin{array}{l}\text { Lisboa-Vale } \\
\text { do Tejo }\end{array}$ & $\begin{array}{l}\text { idepósito? } \\
\text { en poblado }\end{array}$ & Monteagudo, 1977: 212, 1345-134 \\
\hline Castro de Pragança & Pragança & $\begin{array}{l}\text { Estremadura } \\
\text { portuguesa }\end{array}$ & $\begin{array}{l}\text { Lisboa-Vale } \\
\text { do Tejo }\end{array}$ & poblado & Monteagudo, 1977: 212, 1343 \\
\hline Curral das Cabras & Obidos-Columbeira & $\begin{array}{l}\text { Estremadura } \\
\text { portuguesa }\end{array}$ & $\begin{array}{l}\text { Lisboa-Vale } \\
\text { do Tejo }\end{array}$ & ¿depósito? & Monteagudo, 1977: 212, 1344 \\
\hline & Cesareda, Óbidos & $\begin{array}{l}\text { Estremadura } \\
\text { portuguesa }\end{array}$ & $\begin{array}{l}\text { Lisboa-Vale } \\
\text { do Tejo }\end{array}$ & Monteagudo, 1977: 212, 1344A \\
\hline Cabeço de Moinhos & S. Alcainça, Mafra & $\begin{array}{l}\text { Estremadura } \\
\text { portuguesa }\end{array}$ & $\begin{array}{l}\text { Lisboa-Vale } \\
\text { do Tejo }\end{array}$ & poblado & Monteagudo, 1977: 212, 1342A \\
\hline & ¿Elvas? & Alto Alemtejo & Alemtejo & ¿depósito? & Monteagudo, 1977: 212, 1342 \\
\hline Monte Sa Idda & Decimoputzu & Cagliari & Cerdeña & depósito & $\begin{array}{l}\text { Taramelli, 1921: 22-23, fig. 19, 21 y 22; } \\
\text { Monteagudo, 1977: 140 }\end{array}$ \\
\hline
\end{tabular}

Tab. 6. Distribución de las hachas de talón unifacial con una anilla en la Península Ibérica y Cerdeña. Tipo 36B.

\section{Hachas de talón con dos anillas sin nervadura central. Bronce Final IIIA}

Las hachas del tipo 27B marcan, tras el predominio atlántico del Bronce Final II, una distribución exclusivamente mediterránea, y especial- mente del Sureste, Murcia y Granada, con una prolongación hasta Cuenca (Tab. 7; Fig. 7).

$\mathrm{Su}$ distribución mediterránea viene definida por los hallazgos en Formentera, que presenta el depósito más indicativo sobre su cronología en Can Gallet, junto a 4 hachas de apéndice lateral 


\begin{tabular}{|l|l|l|l|l|l|}
\hline \multicolumn{1}{|c|}{ Yacimiento } & \multicolumn{1}{c|}{ Municipio-comune } & \multicolumn{1}{c|}{ Provincia } & \multicolumn{1}{c|}{ Región } & \multicolumn{1}{c|}{ Tipo } & \multicolumn{1}{c|}{ Bibliografía } \\
\hline & Cardenete & Cuenca & $\begin{array}{l}\text { Castilla-La } \\
\text { Mancha }\end{array}$ & & Monteagudo, 1977: 161, 957 \\
\hline Cerro del Real & Galera & Granada & Andalucía & depósito & Monteagudo, 1977: 161, 958 \\
\hline & Caniles & Granada & Andalucía & & Monteagudo, 1977: 162, 959 \\
\hline & Totana & Murcia & Murcia & & Monteagudo, 1977: 162, 960 \\
\hline Museo Valencia & & Murcia-Almería & Murcia-Andalucía & & Monteagudo, 1977: 162, 961 \\
\hline Can Gallet (3) & Sant Francisco Javier & $\begin{array}{l}\text { Formentera- } \\
\text { Baleares }\end{array}$ & Baleares & depósito & Monteagudo, 1977: 162, 961A-B \\
\hline Forraxi Nioi & Nuragus "Valenza" & Nuoro & Cerdeña & depósito & $\begin{array}{l}\text { Nissardi, 1882: fig. 18/24; Lo Schiavo, 1989: } \\
111-112, \text { fig. 3/8 }\end{array}$ \\
\hline
\end{tabular}

Tab. 7. Distribución de las hachas de talón con dos anillas sin nervadura central en la Península Ibérica, Baleares y Cerdeña. Tipo $27 \mathrm{~B}$.

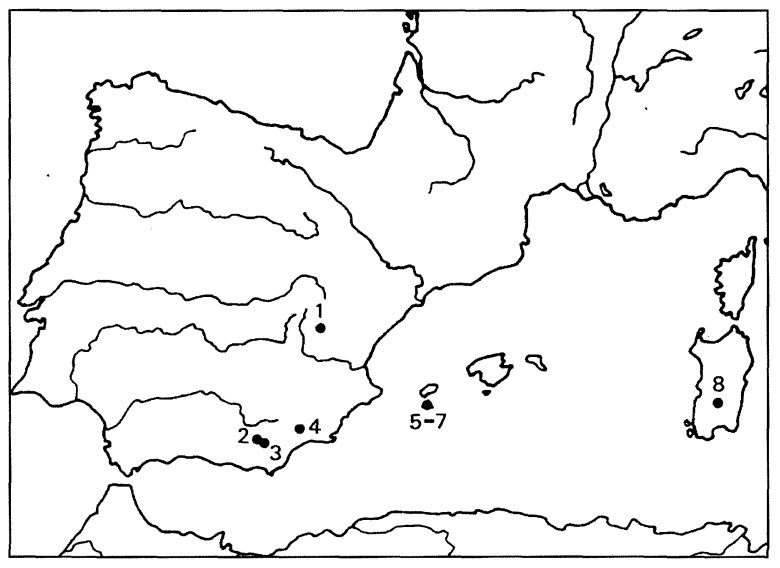

Fig. 7. Distribución de las hachas de talón con dos anillas sin nervadura central en la Península Ibérica, Baleares y Cerdeña. Tipo 27B. 1: Cardenete (Cuenca). 2: Cerro del Real (Granada). 3: Caniles (Granada). 4: Totana (Murcia). 5-7: Can Gallet (Formentera). 8: Forraxi Nioi (Nuoro).

del tipo 22A, las cuales perduran en el Bronce Final IIIB y suelen conservar el "cono de fundición" que reafirma su valor de lingote. El tipo $27 \mathrm{~B}$ tiene su prolongación extrema en el centro-sur de Cerdeña, dentro del depósito de Forraxi Nioi.

\section{Espadas de lengua de carpa tipo Huelva. Bronce Final IIIA}

Estas espadas vinculadas con los momentos iniciales de la metalurgia tipo Huelva, las encon- tramos tanto en Cerdeña, caso de Sequestre, como en el Lazio, San Marinella. El tipo clásico y más evolucionado tambien está presente en el Lazio, Contigiliano (Tab. 8).

Su ausencia en Cataluña, Aragón y País Valenciano, indica dos posibles fuentes de procedencia, Andalucía, o más dudosamente, el litoral atlántico galaico-portugués. La excepción está en los ejemplares del Languedoc y la Provenza (Roudil, 1972: 189, 191; Guilaine et alii, 1991: 308-310), que conectan con otros que se distribuyen a lo largo del valle del Ródano. Existe, por lo tanto, esta tercera posibilidad de procedencia para los cinco ejemplares que conocemos del Mediterráneo Central, aunque el hecho de que al menos dos de ellos se puedan incluir claramente dentro de las espadas de carpa de tipo Huelva favorece la opción ibérica (Fig. 8).

\section{EVOLUCIÓN DE LOS INTERCAMBIOS DE LOS PRODUCTOS METÁLICOS ATLÁNTICOS}

Los datos que hemos analizado creemos que indican con cierta claridad un progresivo desplazamiento de las áreas más dinámicas en el comercio del Bronce Final, desde centros situados en el litoral mediterráneo ibérico hacia el Atlántico, puesto que el Sureste parece mantener cierto predominio durante el Bronce Final IC si nos atenemos a la presencia de espadas Rosnöen (Herrerías en Almería y Llacuna en Barcelona) y de las ha-

\begin{tabular}{|l|l|l|l|l|}
\hline \multicolumn{1}{|c|}{ Yacimiento } & \multicolumn{1}{c|}{ Municipio-comune } & \multicolumn{1}{c|}{ Provincia } & \multicolumn{1}{c|}{ Tipo } & \multicolumn{1}{c|}{ Bibliografía } \\
\hline San Marinella & & Roma, Lazio & depósito & Peroni, 1970: 96-97, taf. 40/269 \\
\hline Contigliano & & Rieti & depósito & Ponzi, 1970: fig. 3 \\
\hline Séquestre & Siniscola & Nuoro & & Lo Schiavo, 1978: 87, fig. 27/1 \\
\hline Castelluccio (2) & & Ragusa, Sicilia & depósito & Di Stefano y Giardino, 1990-91: 509, fig. 15/39-40, 511, 530 \\
\hline
\end{tabular}

Tab. 8. Espadas de lengua de carpa en Italia continental, Cerdeña y Sicilia. 


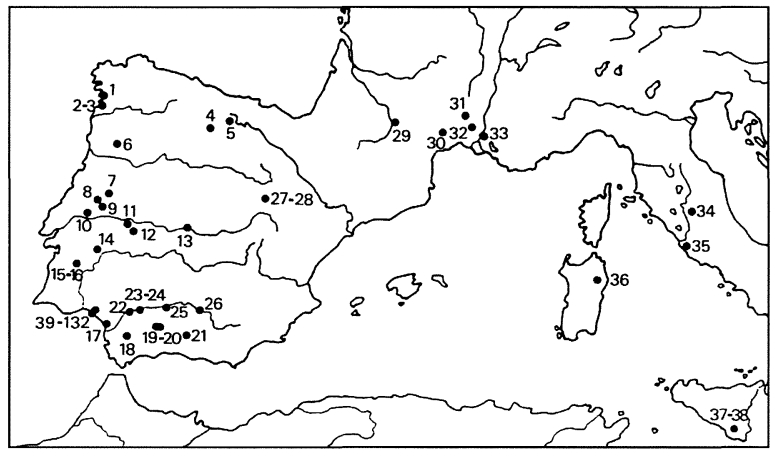

Fig. 8. Distribución de las espadas de lengua de carpa en la Península Ibérica, S. de Francia, Cerdeña, Italia y Sicilia. 1: Covapodre-San Andrés de Hío (Pontevedra). 2-3: Candelas de Tuy-Oleiros (Pontevedra). 4: Peña Amaya (Palencia). 5: Frechilla (Palencia). 6. Val de Támega (Tras-osMontes). 7: Teixoso (Beira Baixa). 8: Castelo Velho de Caratâo (Beira Baixa). 9: Quinta de Erdeval (Beira Baixa). 10: Porto do Concelho (Beira Baixa). 11: Cabezo de Araya (Cáceres). 12: El Risco (Cáceres). 13: Ronda-Carpio del Tajo (Toledo). 14: Elvas (Alto Alemtejo). 15-16: Alemtejo-Safara (Baixo Alemtejo). 17: MatalascañasDoñana (Huelva). 18: Bornos (Cádiz). 19-20: Remanso de las Golondrinas-Los Castellares (Sevilla). 21: Cerro de la Miel (Granada). 22: Corta de la Cartuja-Arroyo Blanco (Sevilla). 2324: La Rinconada-Alcalá del Río (Sevilla). 25: Marmolejo-Villa del Río (Córdoba). 26: Río Guadalimar-Baeza (Jaén). 27-28: Sigüenza (Guadalajara). 29: Vigoulet-Auzil (Haute-Garonne). 30: Octon (Hérault). 31: Barjac (Gard). 32: Sainte-Anastasie (Gard). 33: Tarascon (Bouches-du-Rhône). 34: Contigliano (Rieti). 35: San Marinella (Lazio). 36: Séquestre (Nuoro). 37-38: Castelluccio (Siracusa). 39-132: Ría de Huelva (Huelva).

chas planas de apéndices laterales. El nivel de estos intercambios no debe ser minusvalorado ya que la distribución de estas últimas alcanza ampliamente a Cerdeña, Lazio y Sicilia, en dirección oriental. Pero también se mantiene otra ruta hacia el Oeste, a lo largo del litoral atlántico marroquí, en la ría de Larache, donde está constatada (RuizGálvez, 1983: 65, fig. 1) otra espada Rosnöen.

Por el contrario, parece producirse claramente desde comienzos del Bronce Final II, un desplazamiento de las regiones productoras hacia el litoral cantábrico, ya detectable en los depósitos durante el Bronce Final I. Queda patente en las hachas de talón con sección hexagonal y dos anillas, concentradas en Burgos (Fig. 2), y las espadas pistilifor- mes en Asturias, León y Orense (Fig. 3), mostrando el dinamismo del centro minero asturiano que despega a partir del Calcolítico Medio y Final en las minas de Aramo y El Milagro (Blas Cortina, 1989 y 1992).

Respecto a su producción, la presencia de un molde de espadas pistiliformes en Binefar (Tamarite de Litera, Huesca) (Barril et alii, 1982: 370, fig. 1), es un dato clave, no sólo porque evidencia la fabricación in situ en la Península Ibérica, sino por qué también pone de manifiesto que su elaboración no tiene porque corresponderse con las tradicionalmente denominadas regiones atlánticas. Este yacimiento se emplaza en la ruta que conecta el Garona de la Aquitania francesa, donde existe una masiva presencia de espadas pistiliformes (Coffyn, 1985: carte 16), con la ruta hacia el Valle del Ebro que penetra desde los Pirineos a través del Noguera Ribagorzana, Noguera Pallaresa y Segre (Rovira y Casanovas, 1993: 70, fig. 1, 76). La distribución de las espadas pistiliformes muestra una notoria vinculación continental, y asociación a las rutas de comunicación fluviales y terrestres (Fig. 3).

En Cerdeña, además, varias figuras nurágicas procedentes de Monte Arcosu (Uta, Cagliari) (Lilliu, 1966: 7, 11-12; Thimme, 1980: 105 abb. 75, 116 abb. 85, 268 abb. 90a y b) portan una espada pistiliforme con un notable engrosamiento hacia el centro-tercio final de la mismas, característico de estas espadas, frente a los bordes paralelos de las espadas de lengua de carpa.

Avanzado el Bronce Final II, y ya con claridad a lo largo de todo el Bronce Final IIIA, será la región del litoral atlántico galaico-portugués hasta la margen septentrional del valle del Tajo, Extremadura portuguesa y Beira Baja, a la que cabe atribuir la producción de la mayoría de las hachas conocidas en el Mediterráneo Central, particularmente las de talón y las unifaciales de una o dos anillas.

Esta región ha sido por tales circunstancias presentada como el núcleo más dinámico del Bronce Final III Atlántico (Ruiz-Gálvez, 1984, 1986 y 1993; Coffyn, 1985), sin embargo, queremos introducir un elemento de reflexión. El hecho de que se trate de un área donde se documentan la mayoría de los depósitos, no implica necesariamente que fuera además el área más dinámica, impresión que se obtiene por el volumen de metal que integraba tales depositos, sino que en otras áreas de la Península Ibérica no existían los condicionamien- 
tos sociales que incidieron en la realización deposiciones, ya que otros datos como la distribución de las espadas de lengua de carpa tipo Huelva indican, por el contrario, una mayor demanda por parte de Andalucía.

Si nos atenemos a la distribución de los depósitos durante el Bronce Final III, prácticamente sólo se circunscriben al litoral atlántico entre Galicia y la Península de Setúbal-Beira Baixa (Mederos y Harrison, 1996: fig. 7). Los supuestos depósitos meseteños publicados por Almagro Basch (1940) en Guadalajara y Ocenilla (Soria), podrían corresponderse con poblados, como sucede con el Cabezo de Araya (Cáceres) (Almagro Basch, 1961). Y de los hallazgos del distrito de Beja en el Bajo Alentejo (Schubart, 1975) no conocemos el contexto. En todo caso, si fuesen depósitos, resultan excepcionales.

Por el contrario, si observamos la distribución de las espadas de lengua de carpa (Fig. 8), que consideramos más representativa, este área atlántica sólo cuenta con tres ejemplares en Pontevedra, uno en Tras-os-Montes y cuatro en Beira Baixa, en total ocho, estando totalmente ausentes en regiones costeras como Minho, Douro, Beira Litoral y Extremadura portuguesa, en las cuales, conviene recalcarlo, los porcentajes de distribución de hachas, y particularmente de los tipos que aquí estamos valorando, son muy inferiores a otras comarcas ibéricas interiores, salvo en las hachas de talón unifaciales con una anilla (Fig. 6). Ello contrasta claramente con una región como Andalucía Occidental donde contamos con otras ocho espadas de carpa, a las que se le podrían sumar dos más en Andalucía Oriental, sin necesidad de recurrir a los 93 ejemplares del depósito de Huelva.

Es por ello por lo que, sin prejuzgar el papel que como centros productores desempeñaron las distintas áreas del litoral atlántico portugués, es posible que no sólo fueran dichas regiones las que canalizasen parte de su producción hacia el Mediterráneo Central. Pero tal vez sí transportasen estos artefactos hasta las cercanías del Estrecho de Gibraltar, al estar más acostumbrados a las aguas abiertas del Océano Atlántico, tan distintas del Mediterráneo. Así adquiriría mayor relevancia el depósito o pecio de Huelva como expresión de uno de los potenciales centros redistribuidores hacia el Mediterráneo Central. En todo caso, sólo un pecio bien excavado nos podrá aportar información contextual adecuada.

\section{LA RUTA ORIENTAL. PRODUCCIONES MICÉNICAS EN LA PENÍNSULA IBÉRICA}

La primera evidencia ha surgido con el descubrimiento en 1985 de cerámicas micénicas en el Llanete de los Moros (Montoro, Córdoba) del Heládico Final IIIA2 o IIIB1 (Martín de la Cruz, 1987 y 1988), ca.1375-1225 AC, el cual fue recibido en España con bastante perplejidad y escepticismo tras su publicación.

Las razones de dicha actitud habría que buscarlas en la coincidencia de cuatro circunstancias. $\mathrm{La}$ primera de ellas fue un contexto arqueológico español donde finalmente el paradigma autoctonista comenzaba a consolidarse en los estudios del Calcolítico y Bronce de Andalucía y, a nivel general, en todo el Estado español.

En segundo lugar está la falta de tradición e impacto de la investigadora arqueológica española en el ámbito del Egeo, salvo las continuadas aportaciones de J.L. Melena y M. Ruipérez desde el campo de la linguística, que se hace patente tanto en problemas de formación de especialistas en la Prehistoria Reciente griega, como en la escasez de bibliotecas con la suficiente documentación bibliográfica.

Un tercer factor sería el mínimo conocimiento de Bronce Final I o Bronce Tardío que tenemos en Andalucía de monografías de excavaciones detalladas. Prácticamente sólo disponemos de los poblados de Cuesta del Negro (Purullena, Granada), que incluso mostraba una cerámica completa a torno dentro de niveles del Bronce Final I (Molina González y Pareja, 1975: fig. 102/449), y el Cerro de la Encina (Monachil, Granada) (Arribas et alii, 1974), y en ambos las monografías presentan solamente una selección de cortes.

Finalmente, ha incidido el lento proceso que ha llevado el estudio de dichas piezas desde su descubrimiento hasta la actualidad. En principio, tras mostrarlas en el XI Congreso Internacional de la Unión Internacional de Ciencias Prehistóricas y Protohistóricas (Mainz, 1987), donde pudo consultar a especialistas en cerámica micénica como $\mathrm{K}$. Kilian, antiguo director de las excavaciones de Tirinto, y C. Podzuweit, fueron publicadas bajo el interrogante de ¿cerámicas micénicas en Andalucía? (Martín de la Cruz, 1987). Los datos contextuales del hallazgo serán mostrados un año después con algún detenimiento exclusivamente en alemán (Martín de la Cruz, 1988), y otro tanto sucedió cuando se dispuso de los primeros análisis

T. P., 54, n. $^{\circ} 2,1997$ 
de las mismas, tanto a nivel tipológico (Podzuweit, 1990) como particularmente a nivel analítico (Mommsen et alii, 1990), a pesar de la importancia que tenían para la Península Ibérica, lo que quizás hubiera requerido el esfuerzo de publicarlos traducidos.

Obviamente, Martín de la Cruz ha ido aportando distintos datos sobre estos informes, pero quizás no con suficiente detalle. Por ejemplo, su artículo en alemán de 1988 está publicado en castellano exactamente igual, salvo cuando se realiza la descripción de los contextos de los cortes R-2 y R-3 (Martín de la Cruz, 1984-85: 210-213, fig. $2 / 91$ y $3 / 126$ ). Finalmente, la primera datación del estrato asignado ha tardado tres años más en ser divulgada (Martín de la Cruz y Perlines, 1993). Por lo tanto, aún admitiendo la lógica prudencia que requiere la publicación de hallazgos de esta importancia, dado su carácter precursor, en ocasiones la complejidad y lentitud del proceso no favorece una aceptación mayoritaria.

Remitiéndonos al contexto de las cerámicas (Martín de la Cruz, 1988; Martín de la Cruz y Perlines, 1993), y a la espera de una detallada exposición de la estratigrafía del Corte R-3 en relación con la general del poblado, se documentó en una fosa, que correspondería al nivel III, la cual corta el nivel II precedente. Dicha fosa, se divide en 4 subniveles, pero como no se presentan los subniveles divididos en un perfil dibujado, desconocemos si se tratan de niveles naturales, lo que parece presumible en la subdivisión, o de alzadas artificiales de la excavación.

El primero de los subniveles, III.1, muestra cerámicas con decoraciones excisas y de boquique. En III.2 un fragmento micénico acompaña a boquique. En III.3 continúa el boquique. Y finalmente en III.4, junto a dicha cerámica decorada aparece el segundo fragmento micénico. Del nivel IV sólo disponemos de una pieza dibujada decorada incisa, presumiblemente tipo Cogotas, sobre el que se dispone un derrumbe o nivel V. Las dataciones son CSIC-795 1430 (1310) 1123 AC para "el nivel en el que aparecieron las cerámicas" o III.2/III.4 y CSIC-794 1410 (1260) 1043 AC para el nivel "inmediatamente encima dentro del estrato III" o III.3/III.1. En todo caso, ambas fechas se corresponden bien con el Heládico Final IIIA2 o IIIB, ca.1375-1225 AC, a los que tipológicamente se asignan las piezas.

Es importante recalcar que, aunque Martín de la Cruz (1984-85: 213) señala la presencia de "frag- mentos de campaniforme en el estrato III del corte R-3, asociados en algún caso con las cerámicas micénicas", si nos atenemos a las dibujos publicados, en ningún momento se presenta una auténtica cerámica campaniforme, tratándose de cerámicas decoradas tipo Cogotas. La única excepción en las figuras 2 y 3 es la pieza $2 / 66$, asignable a una cazuela campaniforme, y procede del nivel Ic.

Por otra parte, el argumento de Aubert (1992: 17-18) según el cual estas cerámicas habrían sido importadas a la Península Ibérica en una fecha posterior a la de su fabricación, a partir del 1100 a.C., bajo el supuesto de que ningún dato sugiere intercambios comerciales con el Egeo, no tiene en cuenta las dataciones ya disponibles.

En resumen, como puede observarse, han interactuado cuatro factores de forma negativa, el paradigma epistemológico autoctonista vigente, la falta de especialistas en Prehistoria Reciente del Egeo, el escaso conocimiento que poseemos del Bronce Final I-II que impide contextualizar adecuadamente los hallazgos del Llanete de los Moros, y finalmente, la insuficiente documentación contextual fruto del lento proceso de publicación.

\section{INTERACCIÓN DEL COMERCIO ATLÁNTICO Y MICÉNICO EN EL MEDITERRÁNEO}

Nuestro objetivo final será articular una nueva propuesta de correlación cronológica del Mediterráneo durante el Bronce Final (Tab. 9) y, simultáneamente, ofrecer una explicación que otorgue una correcta dimensión a la interrelación comercial que existió entre el Mediterráneo Occidental y el Mediterráneo Oriental alrededor de las islas del Mediterráneo Central. En este modelo interpretativo se persigue que las presencias aisladas pierdan el carácter exótico que habitualmente se les concede, caso de la cerámica micénica en Andalucía o de productos atlánticos en Cerdeña, Sicilia e Italia continental, para integrarlas dentro de un marco histórico con motivaciones comerciales específicas e intercambios bidireccionales a lo largo de todo el Mediterráneo.

\section{Heládico Final I-II/Bronce Final IA-IB (1625-1425 AC)}

Durante el Heládico Final I-II se produce un incremento de los intercambios en el Mediterráneo 


\begin{tabular}{|c|c|c|c|c|c|c|c|c|}
\hline \multicolumn{3}{|c|}{ GRECIA } & \multicolumn{2}{|c|}{ SICILIA-EOLIAS } & \multicolumn{2}{|c|}{ CERDEÑA } & \multicolumn{2}{|c|}{ PENÍNSULA IBERICA } \\
\hline H.F. I & $\begin{array}{l}1680- \\
1600 / 1580\end{array}$ & $\begin{array}{l}1600- \\
1510 / 05\end{array}$ & $\begin{array}{l}\text { Castelluccio } \\
\text { III-Capo } \\
\text { Graziano IIA }\end{array}$ & $1625 / 1600$ & Nurágico I & $1600-$ & BF IA & $1625-1525$ \\
\hline HF IIA & $\begin{array}{l}1600 / 1580 \\
1520 / 1480\end{array}$ & $\begin{array}{l}1510 / 05- \\
1440\end{array}$ & & & & & BF IB & $1525-$ \\
\hline HF IIB & $\begin{array}{l}1520 / 1480- \\
1445 / 15\end{array}$ & $\begin{array}{l}1440- \\
+1390\end{array}$ & & -1425 & & & & -1425 \\
\hline HF IIIA1 & $1445 / 15$ & $\begin{array}{l}+1390- \\
1370 / 60\end{array}$ & $\begin{array}{l}\text { Castelluccio } \\
\text { IV-Capo } \\
\text { Graziano IIB }\end{array}$ & $1425-$ & & & BF IC & $1425-$ \\
\hline HF IIIA2 & -1320 & $\begin{array}{l}1370 / 60- \\
1340 / 30\end{array}$ & & -1325 & & -1300 & & -1325 \\
\hline HF IIIB & $1320-1225$ & $\begin{array}{l}1340 / 30- \\
1185 / 80\end{array}$ & $\begin{array}{l}\text { Thapsos } \\
\text { Milazzese }\end{array}$ & $1325-1185$ & Nurágico II & $1300-$ & BF IIA/ C.U. I & $1325 / 1300-1225$ \\
\hline HF IIIC1 & $1225-$ & $\begin{array}{l}1185 / 80- \\
1150 / 40\end{array}$ & $\begin{array}{l}\text { Pantálica I } \\
\text { Ausonio I }\end{array}$ & $1185-$ & & -1150 & BF IIB & $1225-1150$ \\
\hline HF IIIC2 & & $\begin{array}{l}1150 / 40- \\
¿ 1100 / 090 ?\end{array}$ & & -1100 & Nurágico III & $1150-$ & $\begin{array}{l}\text { BF IIC Hío- } \\
\text { Baiões }\end{array}$ & $1150-$ \\
\hline HF IIIC3 & -1125 & $\begin{array}{l}1100 / 1090- \\
1065\end{array}$ & $\begin{array}{l}\text { Pantálica II } \\
\text { Ausonio II }\end{array}$ & 1100 & & & & -1050 \\
\hline $\begin{array}{l}\text { Protogeo- } \\
\text { métrico- } \\
\text { submicénico }\end{array}$ & $\begin{array}{l}1125- \\
\text { antes } 995\end{array}$ & $1065-1015$ & & -925 & & . & BF IIIA Huelva & $1050-950 / 925$ \\
\hline Geométrico & antes $995-$ & & Pantálica III & 950/925- & & -850 & $\begin{array}{l}\text { BFIIIB Vénat/ } \\
\text { HIERRO I }\end{array}$ & $950 / 925-875$ \\
\hline
\end{tabular}

Tab. 9. $\mathrm{HF}=$ Heládico Final; $\mathrm{BF}=$ Bronce Final; $\mathrm{CU}=$ Campos de Urnas ibéricos. Dataciones del Egeo según Manning y Weninger, 1992: 648 y Manning, 1995: 217, la primera columna y Warren y Hankey, 1989: 168-169 la segunda, ambas para el Heládico Final. Tykot, 1994: 129 para el Nurágico. Península Ibérica según Mederos, 1995a: 86 y 1996: 98. Sicilia-Eolias, Mederos inédito. Excepto Warren y Hankey, dataciones absolutas calibradas según Stuiver y Reimer, 1993. cal. 3.0.3. Hay dos determinaciones que marcan el final de Milazzese en la acrópolis de Lípari R-365 1257 (1045) 920 AC y la fase de destrucción de Thapsos en Madre Chiesa (Sicilia) A-4731 1381 (1040) 836 AC. El problema es cómo justificar la coexistencia en Ausonio I y II de cerámicas micénicas desde el HF IIIB-IIIC, aunque algunas puedan deberse a intrusiones, y en la sepultura 133 de Pantálica de un vaso del HF IIIC1.

Central a lo largo de dos ejes principales, Apulia en el Adriático y las islas Lípari en el Tirrénico. En el primer caso el objetivo final es el acceso a la ruta del ámbar que llega hasta el valle del Po, que durante este periodo aparece por primera vez en Grecia (Harding, 1984: fig. 13) concentrado en el Peloponeso y particularmente en las sepulturas de pozo de Micenas.

El segundo eje comercial, aprovechando las corrientes marinas, hace escala en Cabo Piccolo, en el sur de Calabria y alcanza las islas Eolias tras cruzar el estrecho de Messina. Dos son los datos que resultan reveladores. Los poblados tipo Castelluccio del Sur de Sicilia, aparentemente, no reciben importaciones micénicas con lo que se está siguiendo la ruta más rápida para acceder al estrecho de Messina. En segundo lugar se opta por alcanzar la isla de Vivara en el golfo de Nápoles, que cobra mayor importancia desde el Heládico Final
IIA, caso de Punta d'Alaca (Marazzi y Tusa, 1991: 114-115, 130-133) y la isla de Ischia, en Collina di Castiglione (Taylour, 1958: 8). El acceso a esta isla sólo puede tener dos explicaciones, como parte de una ruta en dirección septentrional para acceder hacia el Lazio y sur de Etruria, o como una escala lógica en función de las corrientes marinas, puesto que ascendiendo ligeramente más al norte, permite aprovechar la corriente de retorno que toca Cerdeña y posteriormente desciende de nuevo hacia las islas Eolias.

La opción de penetrar en la Campania septentrional resulta descartable, ya que de momento existe una ausencia total de importaciones micénicas incluso alrededor del propio golfo de Nápoles. La ruta hacia el Norte también aún carece de evidencias para este periodo. Sin embargo, ambas necesariamente no tuvieron que ser opciones contrapuestas y sí quizás complementarias, pero el 
objetivo último que nosotros creemos reconocer en la isla de Vivara es una función de puente de acceso hacia Cerdeña. No obstante, también en esta última isla, por el momento, carecemos de hallazgos previos al Heládico Final IIIA2.

Incluso, si se intentara retrotraer las hachas de apéndices laterales del tipo $20 \mathrm{~A}$ a la fase final del Bronce Final IB, la distribución que ofrecen en el S.SO. y N.NO. de Cerdeña (Fig. 1), y el Lazio, Monte Rovello (Montelius, 1895: lám. 121/26), territorios donde aún no ha llegado la cerámica micénica, advierte claramente que falta aún una conexión de dos ámbitos comerciales analizados, el micénico del Mediterráneo Oriental y el atlántico del Mediterráneo Occidental.

\section{Heládico Final IIIA-IIIB/Bronce Final IC- Bronce Final IIA (1425-1225 AC)}

Durante el Heládico Final IIIA-IIIB se mantiene la ruta del ámbar a lo largo del Adriático con hallazgos a lo largo de las islas de mar Jónico, Cefalenia durante el Heládico Final IIIA, Ítaca en el Heládico Final IIIB (Harding, 1984: 83, fig. 2021) y la Italia continental con evidencias de cerámicas micénicas en la Marchie-Marcas, caso de Treazzano di Monsampolo (Lollini, 1982: 197199). Sin embargo, la ruta hacia el mar Tirrénico parece que se potencia. Los asentamientos alrededor del golfo de Taranto se multiplican y en las islas Eolias se mantiene una presencia altamente significativa.

Un problema surge con Vivara donde parece que la presencia de cerámicas micénicas no continúa a lo largo del Heládico Final IIIA2-IIIB. Tal vez otro asentamiento de la costa tirrénica, quizás en el norte de Campania, está realizando su función. Otra posible explicación para Vivara sería defender que las rutas hacia el Norte o hacia Cerdeña hubieran perdido su importancia. Sin embargo, los datos disponibles no apoyan tal punto de vista. En primer lugar, contamos ya con evidencias para la Toscana del Heládico Final IIIA2/B y IIIB en Luni sul Mignone (Östenberg, 1967: 143144) y del Heládico Final IIIB-IIIC1 en Casale Nuovo, Lazio (Angle et alii, 1993; Vagnetti y Jones, 1993: 211-212). En segundo lugar, la ruta hacia el Norte, desde Lipari, parece que se mantiene, dada la continuada presencia de cerámica micénica en su acrópolis (Taylour, 1958 y 1980).

Finalmente, aparece por primera vez cerámica micénica en Cerdeña durante el Heládico Final IIIA2 en Nuraghe Arrubiu (Lo Schiavo, 1992: 178-179, fig. 27), Heládico Final IIIB1 en Nuraghe Antigori (Ferrarese Ceruti et alii, 1987: 16-21) y Heládico Final IIIB en Orosei (Lo Schiavo y Vagnetti, 1980: 371-391), aunque es presumible encontrar en el futuro evidencias más antiguas. El trayecto de las corrientes marinas sigue precisamente esos puntos, siendo el Golfo de Orosei el más adecuado para alcanzar la isla desde la Italia continental. A su alrededor se localizan cerámicas micénicas, y lo que es más significativo, todas las espadas pistiliformes de Cerdeña, asignables al Bronce Final II.

No menos reveladora es la cerámica micénica en Nuraghe Antigori, pues implica que, en vez de partir desde el golfo de Orosei, en dirección hacia el Norte de Sicilia-Lípari, se descendía costeando la isla y, tras alcanzar el golfo de Cagliari, donde se sitúa Nuraghe Antigori, se tomaba como ruta de retorno, la corriente que va a lo largo del Sur de Sicilia. Ésto explica la aparición de cerámica micénica en varios asentamientos durante este periodo desde Agriento (Taylour, 1958: 63-64) hasta Cozzo del Pantano (Orsi, 1893: 9) y ocasionalmente en Malta (Taylour, 1958: 79; Vagnetti, 1970: 361). El que las hachas con apéndices laterales estén particularmente concentradas en el extremo sureste de la isla, alrededor de Siracusa y Catania (Orsi, 1898 y 1900; Di Stefano, 1984; Albanese, 1986-87 y 1989; Di Stefano y Giardino, 1990-91) (Tabla 1) y las hachas de talón con una o dos anillas alcancen el área de Siracusa (Bernabó Brea, 1957 y 1958; Di Stefano, 1984; Di Stefano y Giardino, 1990-91) (Tablas 4 y 5) indican que los productos atlánticos están siguiendo la misma ruta.

Otro tanto cabe decir de la ruta septentrional en dirección hacia el Lazio y Sur de Etruria, que parece abrirse a la cerámica micénica durante el Heládico Final IIIA2/B, de acuerdo con Luni sul Mignone (Östenberg, 1967: 143-144). El hacha de apéndices laterales en Monte Rovello (Montelius, 1895: lám. 121/26), y el hacha de talón con una anilla del depósito de Tolfa (Peroni, 1961: 1.3.5), ambos en el Lazio, indican similar camino para otros productos atlánticos.

Finalmente, el dato más revelador de la expansión y convergencia de las redes comerciales mediterráneas (Fig. 9) es la coincidencia entre la primera aparición de las cerámicas micénicas del Heládico Final IIIA2 en Nuraghe Arrubiu, Cerde-

T. P., 54, n. ${ }^{\circ} 2,1997$ 


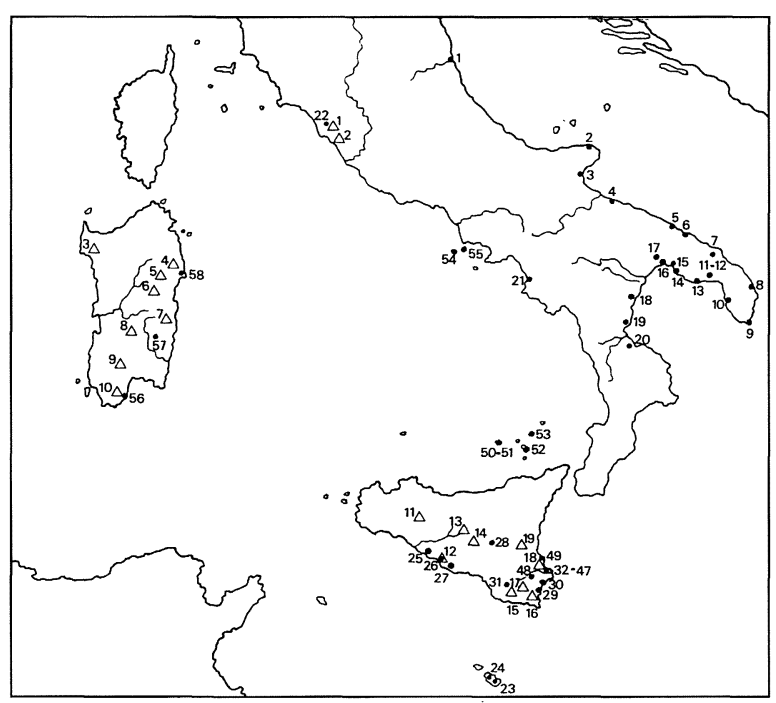

Fig. 9. Convergencia de las redes comerciales atlánticas (hachas de apéndices laterales, hachas de talón con 1 y 2 anillas, espadas pistiliformes) y micénicas (cerámicas) en el Mediterráneo Central durante el Bronce Final IC-IIA y el Heládico Final IIIA-IIIB (1425-1225 AC).

Puntos: cerámica micénica: Treazzano di Monsampolo (Marche). 2: Grotta Manaccora (Apulia). 3: Coppa Nevigata (Apulia). 4: Capo Colonna. Trani (Apulia). 5: Torre Santa Sabina (Apulia). 6: Punta le Terrare. Porto Brindisi (Apulia). 7: San Cosimo d'Oria (Apulia). 8: Via di Uggiano. Otranto (Apulia). 9: Punta Meliso. Santa María di Leuca (Apulia). 10: Scalo di Furno-Porto Cesareo (Apulia). 11: Caverna dell'erba. Avetrana (Apulia). 12: Grotta San Martico. Avetrana (Apulia). 13: Torre Castelluccia (Apulia). 14: Porto Perone (Apulia). 15: Porto Saturo-Satyrion (Apulia). 16: Scoglio del Tonno (Apulia). 17: Cozzo Marziotta (Apulia). 18: Termitito (Basilicata). 19: Broglio di Trebisacce (Calabria). 20: Torre del Mordillo (Calabria). 21: Paestum (Campania). 22: Luni sul Mignone (Lazio). 23: Borg en Nadur (Malta). 24: Tas Silg (Malta). 25: Scirinda (Agriento, Sicilia). 26: Agriento (Sicilia). 27: Madre Chiesa (Agriento, Sicilia). 28: Serra Orlando. Morgantina (Enna, Sicilia). 29: Cozzo del Pantano. sep. 7 (Sicilia). 30: Matrensa-Milocca, sep. 1 (Sicilia). 31: Buscemi (Sicilia). 32: Thapsos. sep. 1 (Sicilia). 33: Thapsos. sep. 2 (Sicilia). 34: Thapsos. sep. 7 (Sicilia). 35: Thapsos. sep. 10 (Sicilia). 36: Thapsos. sep. 14 (Sicilia). 37: Thapsos. sep. 27 (Sicilia). 38: Thapsos. sep. 28 (Sicilia). 39: Thapsos. sep. 37 (Sicilia). 40: Thapsos. sep. 48 (Sicilia). 41: Thapsos. sep. 53 (Sicilia). 42: Thapsos. sep. 61 (Sicilia). 43: Thapsos. sep. 63 (Sicilia). 44: Thapsos. sep. 64 (Sicilia). 45: Thapsos. sep. 1951 (Sicilia). 46: Thapsos. sep. A1 (Sicilia). 47: Thapsos. sep. D (Sicilia). 48: Floridia, sep. 1 (Sicilia). 49: Molinello, sep. 5 (Sici- lia). 50: Capo Graziano. Filicudi (Eolias). 51: Capo Graziano subacuático. Filicudi (Eolias). 52: Acrópolis de Lípari (Eolias). 53: Milazzese. Panarea (Eolias). 54: Collina di Castiglione. Ischia (Pelásgicas). 55: Punta Capitello. Vivara (Pelásgicas) 56: Nuraghe Antigori (Cerdeña). 57: Nuraghe Arrubiu (Cerdeña). 58: Orosei (Cerdeña).

Triángulos: productos atlánticos: 1: Massiccio della Tolfa (Lazio). 2: Monte Rovello (Lazio). 3: Nuraghe Flumenelongu (Cerdeña). 4: Oroè (Cerdeña). 5: Su Tempiesu (Cerdeña). 6: Bolòtana (Cerdeña). 7: Ogliastra (Cerdeña). 8: Albini (Cerdeña). 9: Monte Sa Idda (Cerdeña). 10: Monte Arrubiu (Cerdeña). 11: Polizzello (Sicilia). 12: Agriento (Sicilia). 13: Niscemi (Sicilia). 14: Piazza Armerina (Sicilia). 15: Modica (Sicilia). 16: Noto Antica (Sicilia). 17: Castelluccio (Sicilia). 18: Siracusa (Sicilia). 19: Paterno (Sicilia).

ña (Lo Schiavo, 1992: 178-179, fig. 27), y los primeros fragmentos de cerámica micénica en el Sur de la Península Ibérica, caso del Llanete de los Moros, durante el Heládico Final IIIA2/IIIB1 (Martín de la Cruz, 1988: 84-88, abb. 4/91 y 5/126 y 1990: 50-51, abb. 2; Podzuweit, 1990: 53-56), poniendo en evidencia el papel preponderante jugado por Cerdeña y el S.SE. de Sicilia.

\section{Heládico Final IIIC/Bronce Final IIB-IIC (1225-1050 AC)}

En el Heládico Final IIIC se producen cambios significativos en este proceso de interrelaciones comerciales. La ruta del ámbar desde el Heládico Final IIIB parece que alcanza una mayor distribución en Grecia (Harding, 1984: 83, fig. 21), y ya hay constancia de cerámica micénica incluso en el valle del Po, caso de Fratessina (Bietti Sestieri, 1981: 146) y Fondo Paviani (Vagnetti, 1982c: 208).

La ruta a lo largo del golfo de Tarento se mantiene a juzgar por el número de presencias de cerámica micénica, e incluso hay por primera vez una notable penetración dentro del territorio contiental de la Apulia, Toppo Daguzzo (Cipolloni Sampò, 1982: 102), o la Campania, como la Grotta di Polla (Gastaldi y D’Agostino, 1982: 155-159), y se generalizan las imitaciones de cerámicas micénicas en la Italia meridional.

En Sicilia, Bietti Sestieri (1988: 44-45, 49) plantea una práctica asimilación entre Pantálica y el Egeo, en forma de una "relación formal económica y política" con algunos de los centros 
postmicénicos, por las afinidades en las formas cerámicas, sepulturas artificiales y artefactos metálicos. Sin embargo, no deja de llamar la atención la contradicción que supone que, de entre unas 5.000 tumbas que conocemos en el yacimiento de Pantalica, ciertamente muchas saqueadas, sólo se cuente con un vaso micénico (Vagnetti, 1968: 133). Por otra parte, los escasos ejemplos de cerámicas micénicas corresponden a la fase más antigua, Heládico Final IIIC1 en la sepultura 133 de Pantálica (Vagnetti, 1970: 373) o genéricamente Heládico Final IIIC en Serra Orlando-Morgantina (Sjöqvist, 1960: 134).

Para las Lípari, la destrucción de los asentamientos del Milazzese desde un principio ha sido interpretada por Bernabò Brea (1957) como fruto de la invasión de los Ausonios que aportarían el Subapenínico de la Italia meridional. En todo caso las evidencias de cerámica micénica se rarifican, y se concentran ya sólo en la isla de Lípari.

Otro tanto cabe decir de Vivara, que sigue sin estar ocupada, aunque existe algún yacimiento costero en la Calabria, como Paestum (Kilian, 1969: 345, abb. 6/69-70) con cerámicas de la fase inicial o Heládico Final IIIC1.

La conclusión que cabe obtener es que tanto la ruta de entrada a través del estrecho de Messina, vía las islas Eolias, como la de retorno desde Cerdeña por el sur de Sicilia, se hacen menos frecuentes. En este sentido, no debe olvidarse el impacto negativo que tuvo en el comercio del Mediterráneo Oriental las invasiones de los Pueblos del Mar, ca. 1200/1150-1050 AC que provocó una clara contracción de las redes comerciales (Mederos, 1996: 108-110).

La metalurgia atlántica nos da similar información, y así las hachas de talón unifaciales del Bronce Final IIC no se constatan en el sur de Italia o Sicilia. La situación parece continuar durante el Bronce Final IIIA, caracterizado por las espadas tipo Huelva, o el Bronce Final IIIB y sus típicas hachas tubulares, que tampoco se documentan en dichas regiones meridionales durante los periodos que corresponderían al Protogeométrico y Geométrico Egeo.

Cerdeña, por el contrario, parece mantener el contacto inicialmente, quizás a una escala más reducida, como demuestra la espada tipo Huelva de Séquestre (Lo Schiavo, 1978: 87, fig. 27/1), con emplazamiento alrededor del golfo de Orosei, tal como sucediera con las espadas pistiliformes. Otro tanto cabe pensar para el Lazio de acuerdo con la espada de San Marinella (Peroni, 1970: 9697, taf. 40/269).

Estos datos y la presencia de tres yacimientos de la Etruria meridional, actual Lazio, con cerámica micénica del Heládico Final IIIC, Luni sul Mignone (Östenberg, 1967: 128, 141-142), Monte Rovello (Vagnetti, 1982a: 191; HF IIIB/C) y San Giovenale (Vagnetti, 1982b: 194), advierten que la aparente recesión del comercio micénico alrededor del estrecho de Messina, tiene parte de su explicación en la génesis en Etruria de un centro más dinámico que las regiones meridionales de la Península Italiana y Sicilia, simultáneamente con el predominio continental de los Campos de Urnas, que intensificará las rutas Sur-Norte continentales en detrimento del tradicional eje mediterráneo Este-Oeste.

\section{AGRADECIMIENTOS}

Este trabajo se inició en el Departament of Archaeology, University of Bristol y se culminó en el Institute of Archaeology, Oxford University. Queremos agradecer el apoyo y comentarios de R.J. Harrison y B. Cunliffe. El análisis de la presencia de cerámicas micénicas en la Península Ibérica siempre nos fue incentivado por P. Warren.

\section{BIBLIOGRAFÍA}

Albanese Procelli, R.M. (1986-87): "Un ripostiglio di bronzi da Noto Antica". Atti e Memorie Istituto per lo Studio e la valorizzazione di Noto Antica, 17-18: 45-72.

- (1989): "Asce ad appendici laterali del Museo Archeologico di Siracusa". Archivio Storio per la Sicilia Orientale, 85(1-3): 185-203.

Almagro Basch, M. (1940): "El Hallazgo de la Ría de Huelva y el Final de la Edad del Bronce en el Occidente de Europa". Ampurias, 2: 85-143.

- (1954): "Hacha de bronce de apéndices laterales". Memorias de los Museos Arqueológicos Provinciales, 15: 27.

- (1958): Depósito de la Ría de Huelva. Inventaria Archaeologica. España. Fascículo 1-4. E-1. Instituto Español de Prehistoria-Dirección General de Bellas Artes. Madrid.

- (1961): "El depósito del Bronce III Hispano de Cabezo de Araya. Arroyo de la Luz (Cáceres)". Revista de Estudios Extremeños, 17(1): 5-26.

Almagro Gorbea, M. (1977): El Bronce Final y el Periodo Orientalizante en Extremadura. Bibliotheca Praehistórica Hispana, XIV. Instituto Español de Prehistoria del C.S.I.C. Universidad de Valencia. Madrid. 
- (1996): "El depósito de hachas de Osuna (Sevilla)". Archäologisches Korrespondenzblatt, 26(3): 269-279.

Angle, M.; Conti, A.M.; Dottarelli, R.; Gianni, A. y Persiani, C. (1993): "Prime testimonianze micenee nel Latium Vetus". La Parola del Passato, 48: 190210.

Arribas, A.; Pareja, E.; Molina Fajardo, E..; Arteaga, O. y Molina GonzÁlez, F. (1974): Excavaciones en el poblado de la Edad del Bronce 'Cerro de la Encina'. Monachil (Granada). (El corte estratigráfico $n^{\circ} 3$ ). Excavaciones Arqueológicas en España, 81. Ministerio de Educación y Ciencia. Madrid.

Aubert, C. (1992): "La période pré-phénicienne en Péninsule Ibérique: relations avec la Méditerranée Centrale". Mélanges de la Casa de Velázquez, 28(1): 7-18.

Barril, M.; Delibes, G. y Ruiz Zapatero, G. (1982): "Moldes de fundición del Bronce Final procedentes de 'El Regal del Pídola' (Huesca)". Trabajos de Prehistoria, 39: 369-383.

Bernabò Brea, L. (1957): Sicily before the Greeks. Thames \& Hudson. London.

Bernabò Brea, L. y Cavalier, M. (1960): Meligunis Lipára I. La stazione preistorica della contrada Diana e la necropoli protostorica di Lipari. Publicazioni del $\mathrm{Mu}$ seo Eoliano di Lipari. S.F. Flaccovio Editore. Palermo.

Biancafiore, F. y Toti, O. (1973): Monte Rovello: testimonianze dei micenei nel Lazio. Incunabula Graeca, 53. Edizioni dell' Ateneo. Roma.

Bietti Sestieri, A.M. " (1981): "Economy and Society in Italy between the Late Bronze Age and Early Iron Age”. En G. Barker y R. Hodges (eds.): Archaeology and Italian Society. Prehistoric, Roman and Medieval Studies (Sheffield, 1980). Papers in Italian Archaeology, 2. British Archaeological Reports International Series, 102. Oxford: 133-155.

- (1988): "The 'Mycenaean Connection' and its Impacts on the Central Mediterranean Societies". Dialoghi di Archeologia, 6(1): 23-51.

Blas Cortina, M.A. de (1989): "La minería prehistórica del cobre en las montañas astur-leonesas". En C. Domergue (ed.): Minería y metalurgia en las antiguas civilizaciones mediterráneas y europeas (Madrid, 1985). I. Ministerio de Cultura. Madrid: 143-155.

- (1992): "Minas prehistóricas del Aramo (Riosa). Campaña arqueológica de 1987". Excavaciones Arqueológicas en Asturias, 1987-90: 59-68.

BlinKENBERG, Ch. (1931): Lindos. Fouilles de l'Acropole, 1902-1914. I. Les petits objets. Fondation Carlsberg. Walter de Gruyter. Copnhague-Berlin.

Bosch Gimpera, P. (1945): El poblamiento antiguo y la formación de los pueblos de España. Imprenta Universitaria. México D.F.

Branigan, K. (1974): Aegean Metalwork of the Early and Middle Bronze Age. Clarendon Press. Oxford.

Buchrolz, H.G. y Karageorghis, V. (1971): Altägäis und Altkypros. Verlag Ersnest Wasmuth. Tubingen.
CARANCINI, G.L. (1984): Le asce nell'Italia continentale II. Prähistorische Bronzefunde IX/12. C.H. Beck'sche Verlag. München.

Carrasco, J.; Pachón, J.A. y Pastor, M. (1985): “Nuevos hallazgos en el conjunto arqueológico del Cerro de la Mora. La espada de Lengua de Carpa y la fíbula de codo del Cerro de la Miel (Moraleda de Zafayona, Granada)". Cuadernos de Prehistoria de la Universidad de Granada, 10: 265-333.

Cipolloni Sampò, M. (1982): “Toppo Daguzzo (Rapolla, Potenza)". En L. Vagnetti (ed.): Magna Grecia e Mondo Miceneo. Nuovi documenti. Istituto per la storia e l'archeologia della Magna Grecia. Taranto: 99-102.

Coffyn, A. (1985): Le Bronze Final Atlantique dans la Péninsule Ibérique. Publications du Centre Pierre Paris 11-Collection de la Maison des Pays Ibériques 20. De Boccard. Paris.

Coffyn, A.; Gómez de Soto, J. y Mohen, C. (1981): L'Apogée du Bronze Atlantique. Le dépot de Vénat. L'Age du Bronze en France, 1. Picard. Paris.

DAKARIS, S. (1985): "Epiro e Magna Grecia fino all'età arcaica". Magna Grecia, Epiro e Macedonia (Taranto, 1984). Atti di Convegno di Studi sulla Magna Grecia, 24. Istituto per la Storia e l'Archeologia della Magna Grecia. Taranto: 103-131.

Delibes, G.; Fernández Manzano, J. y Herrán, J.I. (1994): "El depósito del Bronce Final de Pico Cuerno, Sotoscueva (Burgos): sobre la cronología y distribución de las hachas planas con anillas de la Submeseta norte". Boletín de la Institución Fernan González, 73/209: 239-277.

Delibes, G. y Fernández-Miranda, M. (1988): Armas y utensilios de bronce en la Prehistoria de las Islas Baleares. Studia Archaeologica, 78. Universidad de Valladolid. Valladolid.

Deshayes, J. (1960): Les outils de bronze, de l'Indus au Danube (IVe au II $I^{e}$ millénaire). I. Institut Français d'Archéologie de Beyrouth. Bibliothèque archéologique et historique, 71. Paul Geuthner. Paris.

Di Stefano, G. (1984): Castelluccio (Ragusa). Piccola Guida delle stazioni preistorische degli Iblei. Palermo: 94-100.

Di Stefano, G. y Giardino, C. (1990-91): "Sicilia. Ragusa. Il ripostiglio di bronzi in contrada Castelluccio sull'Irminio". Notizie degli Scavi di Antichitá comunicate alla Reale Accademia dei Lincei, $9^{a}$ S., 1-2: 489-546.

DÖRPFELD, W. (1902): Troja und Ilion. Ergebnisse der ausgrabungen in den Vorhistorischen und Historischen schichten von Ilion 1870-1894. Beck \& Barth. Athen.

Esparza Arroyo, A. (1978): "Hacha de apéndices laterales del Castro de Fradellos (Rabanales, Zamora)". Boletín del Seminario de Estudios de Arte y Arqueología, 44: 346-348.

FADDA, M. ${ }^{a} A$. (1988): La fonte sacra di Su Tempiesu. Sar- 
degna Archeologica. Guide e Itinerari, 8. Carlo Delfino Editore. Sassari.

FernándeZ de Avilés y Álvarez Ossorio, A. (1935): “Dos fragmentos interesantes de cerámica incisa procedentes de Murcia”. Boletín del Museo Provincial de Bellas Artes, $\mathrm{s} / \mathrm{n}^{\circ}$.

FERNÁNDEZ GómEZ, J.H. (1973): "Nuevo depósito de hachas de bronce descubierto en la isla de Formentera". Pyrenae, 9: 177-183.

- (1974): "Hachas de bronce halladas en Ibiza y Formentera". VI Symposium de Prehistoria Peninsular (Palma de Mallorca, 1972). Publicaciones Eventuales. Instituto de Arqueología y Prehistoria. Universidad de Barcelona. Barcelona: 63-71.

FERnÁNDEZ MANZANo, J. (1986): Bronce Final en la Meseta Norte española: el utillaje metálico. Investigaciones Arqueológicas en Castilla y León. Monografías. Junta de Castilla y León. Soria.

FernándeZ-Miranda Fernández, M. (1993): "Relaciones exteriores de las Islas Baleares en tiempos prehistóricos". Cuadernos de Arqueología Marítima, 2: 137157.

Ferrarese Ceruti, M.L. (1979): "Ceramica micenea in Sardegna (notizia preliminare)". Rivista di Scienze Preistoriche, 34: 243-253.

Ferrarese Ceruti, M.L; Vagnetti, L. y Lo Schiavo, L. (1987): "Minoici, micenei e ciprioti in Sardegna alla luce delle più recenti scoperte". En M.S. Balmuth (ed.): Studies in Sardinian Archaeology III. Nuragic Sardinia and the Mycenean World. British Archaeological Reports. International Series, 387. Oxford: 7-34.

FröDIN, O. y PERSSON, A.W. (1938): Asine. The results of the Swedish excavations, 1922-1930. Westholm. Stockholm.

Gastaldi, P. y D'Agostino, B. (1982): “Grotta di Polla (Salerno)". En L. Vagnetti (ed.): Magna Grecia $e$ Mondo Miceneo. Nuovi documenti. Istituto per la storia e l'archeologia della Magna Grecia. Taranto: 155159.

George, J. y Chauvet, G. (1894): "Une cachette d'objets en bronze découverte à Venat, commune de SaintYrieux prés d'Angoulême". Bulletin de la Société Archeologique Historique Charente, $6^{\text {a }}$ S., 4: 105-392.

GIARDINO, C. (1995): Il Mediterraneo Occidentale fra XIV ed VIII secolo a.C. Cerchie minerarie e metallurgiche. British Archaeological Reports. International Series, 612. Oxford.

GonZÁLEZ Prats, A. (1985): "Sobre unos elementos materiales del comercio fenicio en tierras del Sudeste peninsular". Lucentum, 4: 97-106.

GuILAINE, J. (1972): L'age du Bronze en Languedoc occidental, Rousillon, Ariége. Mémoires de la Société Préhistorique Française, 9. Klincksieck. Paris.

Guilaine, J.; Briois, F. y Coularou, J. (1991). "L'épée de Trèbes (Aude) et les épées du Sud de la France à l'Age du Bronze Final". En C. Chevillot y A. Coffyn (eds.):
L'Age du Bronze Atlantique (Beynac, 1990). Association des Musées du Sarladais. Beynac-et-Cazenac: 305-311.

HARDING, H. (1975): "Mycenaean Greece and Europe: the Evidence of Bronze Tools and Implements". Proceeding of the Prehistoric Society, 41: 183-202.

- (1984): The Mycenaeans and Europe. Academic Press. London.

HARRISON, R.J. (1974-75): "Nota acerca de algunas espadas del Bronce Final en la Península Ibérica". Ampurias, 36-37: 225-233.

KILIAN, K. (1969): "Neue Funde zur Vorgerschichte Paestums". Mitteilungen des Deutschen Archaeologisches Instituts. Roemische Abteilung, 76: 335-349.

Lilliu, G. (1956): Sculture della Sardegna Nuragica. Della Zattera. Cagliari.

LollinI, D.G. (1982): “Treazzano di Monsampolo (Castel di Lama, Ascoli Piceno)". En L. Vagnetti (ed.): Magna Grecia e Mondo Miceneo. Nuovi documenti. Istituto per la storia e l'archeologia della Magna Grecia. Taranto: 197-199.

LÓPEZ PALOMO, L.A. (1978): "Pequeño depósito de bronces en el río Genil". Cuadernos de Prehistoria de la Universidad de Granada, 3: 233-244.

Lo Schiavo, F. (1976): "Il Ripostiglio del Nuraghe Flumenelongu (Alghero, Sassari). Considerazioni preliminari sul commercio marittimo nel Mediterraneo occidentale in età protostorica". Quaderni della Soprintendenza Archeologica di Sassari e Nuoro, 2: 7-19.

- (1978): "Armi de utensili da Siniscola". Sardegna Centro-Orientale, dal neolitico alla fine del mondo antico. Soprintendenza ai Beni Archeologici per le Provincie di Sassari e Nuoro. Dessì. Sassari: 85-87.

- (1980): "Waffen, werkzeunge und schmuck aus Bronze". En J. Thimme (ed.): Kunst und Kultur Sardiniens vom Neolithikum bis zum Ende der Nuraghenzeit. Verlag C.F. Müller. Karlsruhe: 134-142.

- (1989): "La Sardegna sulle rotte dell'Occidente. I. La Protostoria". Atti di Convegno di Studi sulla Magna Grecia, 29. Istituto per la Storia e l'Archeologia della Magna Grecia. Taranto: 99-161.

- (1991): "La Sardaigne et ses Relations avec le Bronze Final Atlantique". En C. Chevillot y A. Coffyn (eds.): L'Age du Bronze Atlantique (Beynac, 1990). Association des Musées du Sarladais. Beynac-et-Cazenac: 213-226.

- (1992): “Orroli. Nuoro. Nuraghe Arrubiu”. Bollettino di Archeologia, 13-15: 175-181.

Lo Schiavo, F. y Vagnetti, L. (1980): "Micenei in Sardegna?". Rendiconti della Accademia Nazionale dei Lincei. Classe di Scienze morali, storiche e filologiche, $8^{\mathrm{a}}$ S., 35: 371-393.

MAcWhite, E. (1951): Estudios sobre las relaciones atlánticas de la Península Ibérica en la Edad del Bronce. Disertaciones Matritenses, 2. Seminario de Historia Primitiva. Madrid.

T. P., 54, n. $^{\circ} 2,1997$ 
Manning, S.W. (1995): The Absolute Chronology of the Aegean Early Bronze Age. Archaeology, Radiocarbon and History. Monographs in Mediterranean Archaeology, 1. Sheffield Academic Press. Sheffield.

Manning, S.W. y Weninger, B. (1992): "A light in the dark: archaeological wiggle matching and the absolute chronology of the close of the Aegean Late Bronze Age". Antiquity, 66: 636-663.

Marazzi, M. y Tusa, S. (eds.) (1991): "Relazione preliminare sui lavori nell'isola di Vivara negli anni 198687'. Dialogui di Archeologia, $3^{\text {a }}$ S., 9: 111-138.

MARTín DE LA CRUZ, J.C. (1984-85): "Problemas en torno a la definición del Bronce Tardío en la Baja Andalucía". Cuadernos de Prehistoria y Arqueología de la Universidad Autónoma de Madrid, 11-12: 205-215.

- (1987): “¿Cerámicas micénicas en Andalucía?”. Revista de Arqueología, 78: 62-64.

- (1988): "Mykenische Keramik aus bronzezeitlichen Siedlungs schichten von Montoro aus Guadalquivir". Madrider Mittelungen, 29: 77-92.

- (1990): "Die erste mykenische Keramik von der Iberischen Halbinsel". Präehistoriche Zeitschrift, 65: 49-52.

Martín de la Cruz, J.C. y Perlines, Ma . (1993): "La cerámica a torno de los contextos culturales de finales del II milenio A.C. en Andalucía". I Congresso de Arqueología Peninsular (Porto, 1993). II. Porto. Trabalhos de Antropologia e Etnologia, 33(3-4): 335-349.

Maryon, H. (1938): “Some Prehistoric Metalworkers' Tools". The Antiquaries Journal, 18: 243-250.

MAXwell-Hyslop, R. (1953). "Bronze Lugged Axe- or Adze Blades from Asia". Iraq, 15: 69-87.

Mederos Martín, A. (1995a): "La cronología absoluta de la Prehistoria Reciente del Sureste de la Península Ibérica". Pyrenae, 26: 53-90.

- (1995b): “¿Retorno al pasado?. Comercio o difusión en los análisis de los Sistemas Mundiales Antiguos". Trabajos de Prehistoria, 52(2): 131-141.

- (1996): "La conexión levantino-chipriota. Indicios de comercio atlántico con el Mediterráneo Oriental durante el Bronce Final (1150-950 AC)". Trabajos de Prehistoria, 53(2): 95-115.

Mederos, A. y Harrison, R.J. (1996): "Patronazgo y Clientela. Honor, Guerra y Festines en las Relaciones Sociales de Dependencia del Bronce Final Atlántico en la Península Ibérica". Pyrenae, 27: 31-52.

Molina González, F. (1978): "Definición y sistematización del Bronce Tardío y Final en el Sudeste de la Península Ibérica". Cuadernos de Prehistoria de la Universidad de Granada, 3: 159-232.

Molina González, F. y PAREJA, E. (1975): Excavaciones en la Cuesta del Negro (Purullena, Granada). Campaña de 1971. Excavaciones Arqueológicas en España, 86. Ministerio de Educación y Ciencia. Madrid.

Mommsen, H.; Diehl, U.; Lambrecht, D.; Pantenburg, F.J. y WEBER, J. (1990): "Eine mykenische Scherbe in Spanien: Bestätigung iher mit der Neutronenakti- vierungsanalyse (NNA)". Präehistoriche Zeitschrift, 65: 59-61.

Monteagudo García, L. (1977): Die Beile auf der Iberischen Halbinsel. Prähistorische Bronzefunde, IX/6. C.H. Beck'sche Verlag. München.

Montelius, O. (1895): La Civilisation Primitive en Italie depuis de l'introduction des Métaux. I. Italie septentrionale. Imprimerie Royale. Stockholm.

MülleR-KARPE, H. (1959): Beiträge zur Chronologie der Urnenfelderzeit Nördlich und Südlich der Alpen. Römisch-Germanische Forschungen, 22. Walter de Gruyter. Berlin.

NisSARDI, F. (1882): "Nuragus". Notizie degli Scavi di Antichitá comunicate alla Reale Accademia dei Lincei, 2: 305-311.

ORSI, P. (1893): "Necropoli sicula presso Siracusa con vasi e bronzi micenei (Cozzo Pantano)". Reale Accademia dei Lincei, Monumenti Antichi, 2: 5-35.

- (1898): "Notizie Diverse". Bulletino di Paletnologia Italiana, 24: 162-163.

- (1900): "Ripostiglio di bronzi siculi. I. Ripostiglio di Modica (Siracusa)". Bulletino di Paletnologia Italiana, 26: 164-174.

ORTEGO Y FríAs, T. (1957): "Bronce Atlántico en Territorio Soriano". IV Congreso Nacional de Arqueología (Burgos, 1955). Secretaría General de los Congresos Arqueológicos Nacionales. Zaragoza: 113-121.

ÖsTENBERG, C.E. (1967): Luni sul Mignone e problemi della preistoria d'Italia. Acta Instituti Romani Regni Sueciae, 25. Lund.

Pendlebury, H.W.; Pendlebury, J.D.S. y Money Coutts, M.B. (1937-38): "Karphi. A City of Refuge of the Early Iron Age in Crete". The Annual of the British School at Athens, 38: 57-145.

PERONI, R.B. (1961): Ripostiglio dell'età dei metalli. I. Ripostigli del Massiccio della Tolfa. Inventaria Archeologica. Italia. Fascículo I1-I3. Sansoni. Roma.

- (1970): Le spade nell'Italia continentale. Prähistorische Bronzefunde, IV/1. C.H. Beck'sche Verlag. München.

Pesce, G. (1952): "Daghe sarde, $n^{\circ}$ 2144". Fasti Archeologici, 5: 187-188.

PInZA, G. (1901): "Monumenti Primitivi della Sardegna". Reale Accademia dei Lincei. Monumenti Antichi, 11: 6-278.

Podzuweit, C. (1990): "Bemerkungen zur mykenischen Keramik von Llanete de los Moros, Montoro, Prov. Córdoba". Präehistoriche Zeitschrift, 65: 53-58.

Ponzi Bonomi, L. (1970): "Il ripostiglio di Contigliano". Bullettino di Paletnologia Italiana, 79: 95-153.

Ros Sala, M.'M. (1986): “El Bronce Tardío y Final”. En J. Mas (ed.): Historia de Cartagena. II. Primeros poblamientos del Sureste. Ediciones Mediterráneo. Murcia: 317-352.

Roudil, J.L. (1972): L'Age du Bronze en Languedoc Oriental. Mémoires de la Société Préhistorique Française, 10. Paris. 
Rovira, J. y Casanovas, A. (1993): "El depósito de brazaletes de Sant Aleix (Lleida) y los Depósitos de Objetos Metálicos del Bronce Final en Cataluña". Complutum, 4: 69-80.

Ruiz-Gálvez Priego, M. ${ }^{a} L$. (1983): "Espada procedente de la Ría de Larache en el Museo de Berlín Oeste". Homenaje al Prof. Martín Almagro Basch. II. Ministerio de Cultura. Madrid: 63-68.

- (1984): La Península Ibérica y sus relaciones con el círculo cultural atlántico. Universidad Complutense de Madrid. Madrid.

- (1986): "Navegación y comercio entre el Atlántico y el Mediterráneo a fines de la Edad del Bronce". Trabajos de Prehistoria, 43: 9-42.

- (1993): "El Occidente de la Península Ibérica, punto de encuentro entre el Mediterráneo y el Atlántico a fines de la Edad del Bronce". Complutum, 4: 41-68.

- (ed.) (1995): Ritos de Paso y puntos de paso: La ría de Huelva en el mundo del Bronce Final Europeo. Complutum. Extra, 5. Madrid.

Schubart, H. (1975): Die Kultur de Bronzezeit im Südwesten der Iberischen Halbinsel. Madrider Forschungen, 9. Walter de Gruyter. Berlin.

Schubart, H. y Arteaga, O. (1983): "La Cultura de «El Argar». Excavaciones en Fuente Alamo". Revista de Arqueología, 24-25-26: 16-27, 54-63, 56-63.

SIMÓN GARCÍA, J.L. (1995): "Contribución a la metalurgia del Bronce Final en las Comarcas Centrales Valencianas. Las hachas de apéndices laterales". Recerques del Museu d'Alcoi, 4: 177-183.

SiRET y Cels, L. (1913): Questions de chronologie et d'ethnographie iberiques. I. De la fin du Quaternaire a la fin du Bronze. Paul Geuthner. Paris.

SıöQvist, E. (1960): "Excavations at Morgantina (Serra Orlando) 1959: preliminary report 4". American Journal of Archaeology, 64: 125-135.

Stuiver, M. y ReImer, P.J. (1993): "Extended C-14 data base and revised Calib 3.0 C-14 age calibration program". Radiocarbon, 35(1): 215-230.

TARAmelli, A. (1911): "Serri. Ricerche nell' acropoli di S. Vittoria e nel recinto sacro". Notizie degli Scavi di Antichitá comunicate alla Reale Accademia dei Lincei, 32: 291-312.

- (1915): "Decimoputzu (Cagliari). Scoperta di un ripostiglio di bronzi di età preromana a Monte Sa Issa". Notizie degli Scavi di Antichitá comunicate alla Reale Accademia dei Lincei, 37: 89-97.

- (1921): "Il ripostiglio di bronzi nuragici di Monte Sa Idda di Decimoputzu (Cagliari)". Reale Accademia dei Lincei. Monumenti Antichi, 27: 5-98.

- (1926): "Ripostiglio di bronzi nuragici di Monte Arrubiu". Reale Accademia dei Lincei. Monumenti Anti- chi, 31: 446-456.

TAYlour, W. (1958): Mycenaean Pottery in Italy and adjacent Areas. Cambridge University Press. Cambridge.

- (1980): "Aegean sherds found at Lipari". En L. Bernabò Brea y M. Cavalier (eds.): Melingunis Lipára. IV. L'Acropoli di Lipari nella Preistoria. Publicazioni del Museo Eoliano di Lipari. S.F. Flaccovio Editore. Palermo: 791-817.

Thimme, J. (ed.) (1980): Kunst und Kultur Sardiniens vom Neolithikum bis zum Ende der Nuraghenzeit. Verlag C.F. Müller. Karlsruhe.

Түкот, R.H. (1994): "Radiocarbon dating and absolute chronology in Sardinia and Corsica". En R. Skeates y R. Whitehouse (eds.): Radiocarbon Dating and Italian Prehistory. Archaeological Monographs of the British School at Rome, 8-Accordia Specialist Studies on Italy, 3. London: 115-145.

VAGNETTI, L. (1968): "I bacili di bronzo di Caldare sono ciprioti?". Studi Micenei ed Egeo Anatolici, 7: 129138.

- (1970): "I Micenei in Italia: la documentazione archeologica”. La Parola del Passato, 25: 359-380.

- (1982a): "Monte Rovello (Allumiere, Roma)". En L. Vagnetti (ed.): Magna Grecia e Mondo Miceneo. Nuovi documenti. Istituto per la storia e l'archeologia della Magna Grecia. Taranto: 191.

- (1982b): "San Giovenale (Blera, Viterbo)". En L. Vagnetti (ed.): Magna Grecia e Mondo Miceneo. Nuovi documenti. Istituto per la storia e l'archeologia della Magna Grecia. Taranto: 194.

- (1982c): "Fondo Paviani (Torretta di Legnago, Verona)". En L. Vagnetti (ed.): Magna Grecia e Mondo Miceneo. Nuovi documenti. Istituto per la storia e l'archeologia della Magna Grecia. Taranto: 208.

VAGNETTI, L. y JoNES, R.E. (1993): "Le ceramiche di tipo

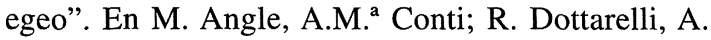
Gianni y C. Persiani (eds.): "Prime testimonianze micenee nel Latium Vetus". La Parola del Passato, 48: 211-213.

Vogel, J.C. Y WATERBOLK, H.T. (1972): "Groningen Radiocarbon Dates X". Radiocarbon, 14(1): 74-75.

Wardle, K.A. (1989): "Excavations at Assiros Toumba 1988. A Preliminary Report". The Annual of the British School at Athens, 84: 447-463.

Warren, P. y Hankey, V. (1989): Aegean Bronze Age Chronology. Bristol Classical Press. Bristol.

Zervos, Ch. (1954): La Civilisation de la Sardaigne. Du debut de l'Énéolithique a la fin de la période nouragique. II ${ }^{e}$ millénaire-Ve siècle avant notre ère. Éditions Cahiers d'Art. Paris. 